A dynamic copula approach to recovering the index implied volatility skew

Matthias R. Fengler, Helmut Herwartz, Christian

Werner

February 2012 Discussion Paper no 2010-33 
Editor: Martina Flockerzi

University of St. Gallen

Department of Economics

Varnbüelstrasse 19

$\mathrm{CH}-9000$ St. Gallen

Phone +41712242325

Fax +41712243135

Email_vwaabtass@unisg.ch

Publisher: Department of Economics

University of St. Gallen

Varnbüelstrasse 19

$\mathrm{CH}-9000$ St. Gallen

Phone +41712242325

Fax +41712243135

Electronic Publication: http://www.vwa.unisg.ch 


\title{
A dynamic copula approach to recovering the index implied volatility
}

$$
\text { skew }^{1}
$$

\author{
Matthias R. Fengler, Helmut Herwartz, Christian Werner
}

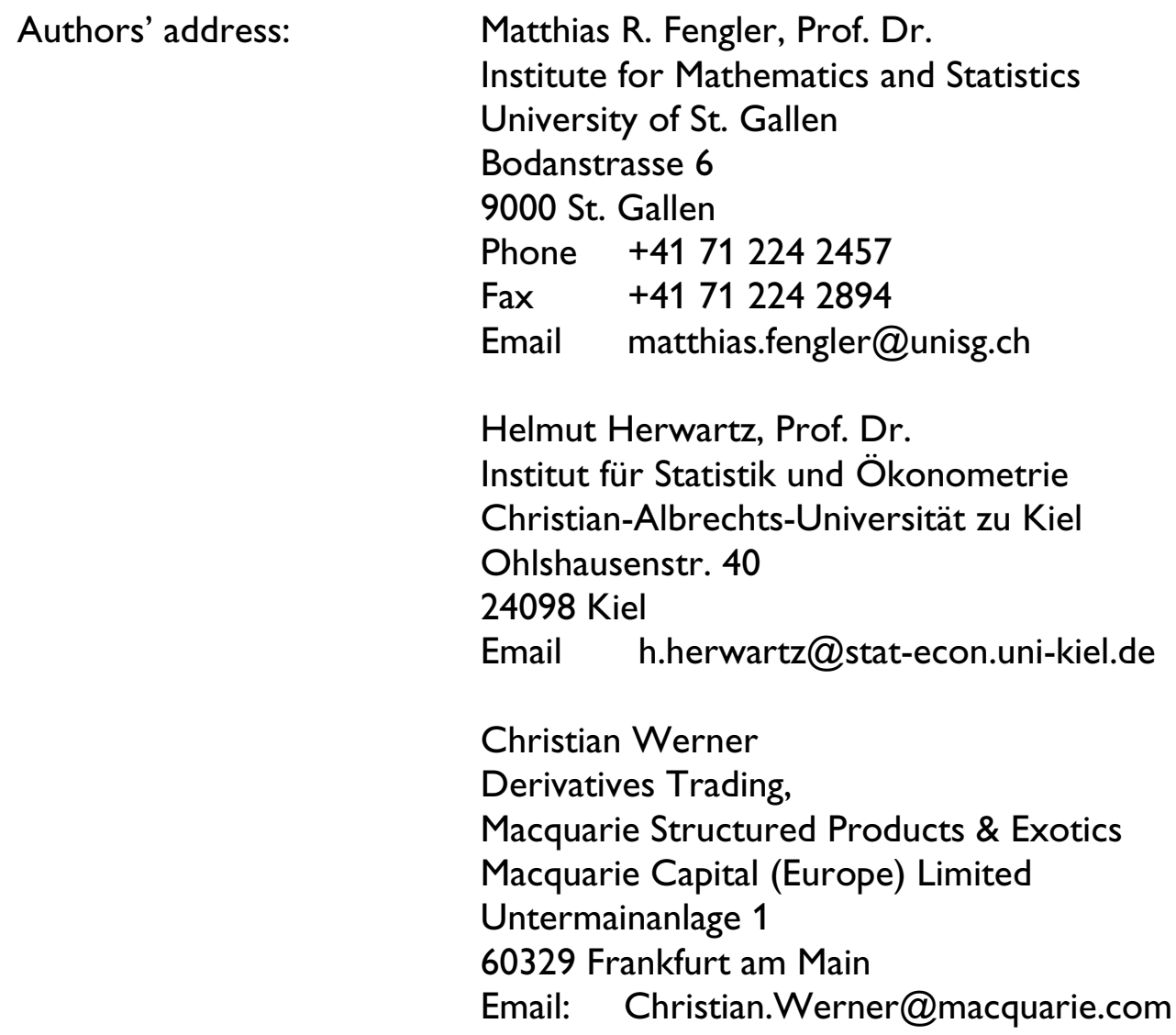

\footnotetext{
${ }^{1}$ We like to thank Jan Kallsen, Christian Menn and Sabrina Mulinacci for comments and stimulating discussions. Earlier versions of this work circulated under the names "Explaining the index skew by means of copula dynamic conditional correlation models" and "Option pricing under time varying correlation with conditional dependence: A copula based approach to recovering the index skew from the constituent dynamics".
} 


\begin{abstract}
Equity index implied volatility functions are known to be excessively skewed in comparison with implied volatility at the single stock level. We study this stylized fact for the case of a major German stock index, the DAX, by recovering index implied volatility from simulating the 30 dimensional return system of all DAX constituents. Option prices are computed after risk neutralization of the multivariate process which is estimated under the physical probability measure. The multivariate models belong to the class of copula asymmetric dynamic conditional correlation models. We show that moderate taildependence coupled with asymmetric correlation response to negative news is essential to explain the index implied volatility skew. Standard dynamic correlation models with zero tail-dependence fail to generate a sufficiently steep implied volatility skew.
\end{abstract}

\title{
Keywords
}

Copula Dynamic Conditional Correlation, Basket Options, Multivariate GARCH Models, Change of Measure, Esscher Transform.

\section{JEL Classification}

C32, C15, G13, G14. 


\section{Introduction}

For equity option markets, it is a widely documented stylized fact that the Black-Scholes implied volatility function is highly asymmetric across strikes ever since the 1987 stock market crash. More precisely, the implied volatility function, which is derived by equating observed option prices at different strikes with the Black and Scholes (1973) option valuation formula, regularly exhibits a steeper slope for out-of-the-money put than for out-of-themoney call options, see e.g. Rubinstein (1994) among many others. This stylized fact, which is usually referred to as the (equity) implied volatility skew, has received a high level of attention in the literature. A second stylized fact of equity option markets, which has attracted much less attention so far, is the observation that individual stock option skews tend to be flatter than the implied volatility skew of the index. For instance, as Bakshi et al. (2003) and Bollen and Whaley (2004) find for U.S. equity option markets and Branger and Schlag (2004) for the German market, the index skew is significantly steeper than the individual implied volatility skews of the constituents. This is surprising, since an index is a mere basket of single stocks by construction. By portfolio diversification effects or from liquidity considerations, one could also expect a reverse pattern.

Several hypotheses have been raised in the literature to explain the counterintuitive relation of index versus stock implied volatility functions. Bakshi et al. (2003) attribute the described stylized volatility features to differences in the skewness of the risk neutral distribution of the index and individual stocks. They propose a market model in which individual stock returns are decomposed into a market and an idiosyncratic factor and derive conditions under which individual skews can be less negatively sloped than the index skew. In contrast, Bollen and Whaley (2004) argue that the steepness of index skews does not result from fundamental differences in the underlying distributions or processes but from supply and demand conditions which drive up put prices. Branger and Schlag (2004) point out that the index being a basket of stocks must be determined from the asset price dynamics of its 
constituents. Using a multivariate jump-diffusion model, they show that excess basket skews can stem from low correlation among the underlying Wiener processes and a high probability of a common downward jump of all stocks. Remarkably, Bollerslev et al. (2008) find strong evidence for non-diversifiable, modest-sized cojumps in individual stocks in high-frequency data. In an empirical asset pricing study, Driessen et al. (2009) further demonstrate that the differential pricing of individual and index options can be attributed to a large correlation risk premium embedded in index options, whereas idiosyncratic variance risk in individual options appears to be unpriced. Similar insights are obtained in Carr and Wu (2009).

In this work we choose a bottom-up approach and model the index as a basket of single stocks. This is accomplished by a full-fledged multivariate model for all constituent stocks comprised in the index. Doing so, we build on extensions of models with generalized autoregressive conditional heteroskedasticity (GARCH), dynamic conditional correlation (DCC, Bollerslev (1990), Engle (2002), Tse and Tsui (2002), Pelletier (2006)) and conditional cross sectional dependence captured by a copula function (Lee and Long 2009). We then ask ( $i$ ) to which extent the model is able to replicate the observed patterns of implied volatility skews in index options; and (ii) which model characteristics govern these features. The model allows us to study the relevance of dynamic correlation and of the joint cross sectional dependence beyond correlation simultaneously. While these questions have frequently been investigated for the univariate case (see Engle and Mustafa (1992), Christoffersen and Jacobs (2004), Christoffersen et al. (2006), Barone-Adesi et al. (2010) amongst others), we address them within a multivariate framework.

The joint analysis of stock return and option data has recently triggered substantial interest in multivariate option pricing models. Langnau (2010) extends the multivariate deterministic volatility model by allowing for a local correlation which depends both on time and the path of the underlying index. Heston-type multivariate option pricing models are suggested by Gouriéroux and Sufana (2004) and da Fonseca et al. (2008), while Luciano and Schoutens (2006) develop a multivariate Lévy model which is driven by a common stochastic 
time change. Factor models are proposed by Mo and Wu (2007), Şerban et al. (2008) and Elkamhi and Ornthanalai (2009) to account for global versus country-specific and market versus firm-specific factors in return and volatility. Unlike this literature we adopt a copula DCC-GARCH model (C-DCC henceforth) due to its tractability in high-dimensions and its flexibility. Moreover, the nested structure of the C-DCC model allows us to disentangle the various sources that potentially contribute to the index skew, namely $(i)$ alternative distributional assumptions on model innovations; (ii) uncorrelated and independent versus uncorrelated and (tail-)dependent innovations; (iii) conditionally heteroskedastic volatility coupled with symmetric versus leveraged conditional correlation dynamics. ${ }^{1}$ Multivariate option pricing in GARCH models is also considered in Goorbergh et al. (2005), Bernard and Czado (2010), and Rombouts and Stentoft (2011) though for much smaller dimensions than considered here.

Adopting a multivariate modeling approach to derivative valuation is challenging from several points of view. First, by the curse of dimensionality, a direct calibration of the model, e.g. by minimizing a cost functional defined across model implied and observed market prices as in Barone-Adesi et al. (2010), does not seem to be viable in a high dimensional model framework. We therefore need to identify model parameters from historical stock price data. To this end, we rely on recent work by Engle et al. (2009) who suggest the use of composite maximum likelihood (CML) estimation, coupled with quasi maximum likelihood (QML) estimation for the marginal variance dynamics. Second, since CML/QML estimates are obtained under the physical probability measure, we cannot compute option prices by means of the estimated model without making very restrictive assumptions on the market price of risk or the underlying distributional framework. Thus, an equivalent risk-neutral pricing measure is called for, under which single stock processes are martingales with respect to the filtration generated by all assets. We therefore move on in applying a feasible change of measure to obtain an equivalent risk-neutral pricing measure. This measure change, which builds on work by Gouriéroux and Monfort (2007) and Christoffersen et al. (2010), requires knowledge of the moment generating function (MGF), which is unknown in our particular 
copula-based model framework. We therefore approximate the change of measure up to second order, which allows us to treat the copula shape parameter as a free parameter. By varying it, we study to which extent the basket implied volatility functions of the riskneutralized model fit the index option market.

Our results are twofold. First, the C-DCC model, when estimated from time series data under the physical measure and risk-neutralized in the manner we outline below, cannot fully account for the observed basket skew. When treating the copula shape parameter as a free variable to search for the best fit, nearly all goodness of fit measures drop by around $30 \%$, but there still remains some degree of steepness in the basket skew which cannot be explained. Thus, the proposed measure change fails to capture all aspects of the risk-neutral measure adopted by the market. Evaluating the out-of-sample pricing performance of the model, we find this outcome to be robust across a couple of subsequent trading days. As a second part of results, a sensitivity analysis suggests that fat-tailedness and the precise dynamic specification of the threshold variance processes are not the decisive determinants of the observed stylized facts of index versus constituent skews. This is because fat-tailedness at the constituent level vanishes to a large extent at the basket level, while an increased response of constituent variances to bad news has a similarly sized impact on both the basket skew and the constituent skew. What substantially matters is the overall dependence structure in the basket, in particular the non-zero probability of large negative returns in all stocks coupled with a strong asymmetric response of the correlation dynamics to bad news, e.g. after joint price deteriorations. These findings corroborate the common factor hypothesis raised for instance in Branger and Schlag (2004) and Driessen et al. (2009), but additionally stress the relevance of particular forms of correlation dynamics to explain the basket skew.

In Section 2, the C-DCC model is presented, and a discussion of model estimation follows in Section 3. In Section 4, we describe the theory for the change of measure in multivariate GARCH models and discuss option pricing. In Section 5 we provide and interpret the empirical results, and Section 6 concludes. 


\section{C-DCC models}

In this section we present the C-DCC model as introduced by Lee and Long (2009). We discuss the variance dynamics of the marginal distributions, the treatment of conditional leptokurtosis, the specification of the correlation dynamics and, finally, the conditional dependence of the data generating processes which drive asset prices.

\subsection{The C-DCC model}

Consider an $N$-dimensional vector of asset returns, $r_{t}=\left(r_{1, t}, \ldots, r_{N, t}\right)^{\top}, r_{i, t}=\log \left(S_{i, t} / S_{i, t-1}\right)$, where $S_{i, t}$ is the price of asset $i$ at time $t$ and assume that first and second order moments of $r_{t}$ are measurable with respect to $\mathcal{F}_{t-1}$, the filtration generated by the multivariate process. The copula multivariate GARCH model reads as

$$
\begin{aligned}
r_{t} & =\mu_{t}-\gamma_{t}+H_{t}^{1 / 2} \varepsilon_{t} \\
\varepsilon_{t} & =\Sigma^{-1 / 2} \eta_{t} \\
\eta_{t} \mid \mathcal{F}_{t-1} & \sim C\left(F_{1}\left(\eta_{1, t} \mid \delta_{1}\right), \ldots, F_{N}\left(\eta_{N, t} \mid \delta_{N}\right), \theta\right),
\end{aligned}
$$

where $\varepsilon_{t}=\left(\varepsilon_{1, t}, \ldots, \varepsilon_{N, t}\right)^{\top}$ and $\eta_{t}=\left(\eta_{1, t}, \ldots, \eta_{N, t}\right)^{\top}$. The vectors $\mu_{t}$ and $\gamma_{t}$ in (1) collect the $\mathcal{F}_{t-1}$-measurable asset specific conditional mean processes and asset specific compensators, respectively, which are explained in more detail below. The conditional covariance matrix of $r_{t}$ is denoted by $H_{t}$, where the matrix square root is implemented by means of the Cholesky decomposition. ${ }^{2}$ Let $\mathrm{E}^{P}\left[X \mid \mathcal{F}_{t-1}\right]$ be the $\mathcal{F}_{t-1^{-}}$-conditional expectation of some random variable $X$ under the physical probability measure $P$, under which the estimation is carried out. For the innovation vectors in $(2)$ it is assumed that $\mathrm{E}^{P}\left[\eta_{t} \mid \mathcal{F}_{t-1}\right]=0$ and $\mathrm{E}^{P}\left[\eta_{t} \eta_{t}^{\top} \mid \mathcal{F}_{t-1}\right]=\Sigma=\left(\sigma_{i j}\right), i, j=1, \ldots, N$. As a consequence the elements in $\varepsilon_{t}$ are uncorrelated processes with mean zero and unit variance, i.e. $\mathrm{E}^{P}\left[\varepsilon_{t} \mid \mathcal{F}_{t-1}\right]=0$ and $\mathrm{E}^{P}\left[\varepsilon_{t} \varepsilon_{t}^{\top} \mid \mathcal{F}_{t-1}\right]=I_{N}$, where $I_{N}$ denotes the $N$-dimensional identity matrix. Although contemporaneously uncorrelated, the elements in $\varepsilon_{t}$ are not independent since they obey a mixture 
representation of the random processes $\eta_{i, t}$. The marginal distributions of $\eta_{i, t}, i=1, \ldots, N$, are denoted by $F\left(\eta_{i, t} \mid \delta_{i}\right)$, and the joint distribution can be written by means of the copula as $C\left(F_{1}\left(\eta_{1, t} \mid \delta_{1}\right), \ldots, F_{N}\left(\eta_{N, t} \mid \delta_{N}\right), \theta\right)$. The parameters $\delta$ and $\theta$ govern the (excess) kurtosis of $\eta_{i, t}$ and the degree of contemporaneous dependence, respectively. Depending on the particular copula, $\theta$ reflects forms of tail-dependence between pairs of assets. For instance, one could capture the notion that a simultaneous price deterioration between pairs of assets is a-priori more likely than a joint upward movement of prices. In particular, if $C(\ldots, \theta)$ is the Gaussian copula and $F\left(\eta_{i, t} \mid \delta_{i}\right)$ the Gaussian distribution, the model coincides with common multivariate GARCH specifications formalized within a framework of conditional normality. In the following, the basic building blocks of the C-DCC model are sketched.

\subsection{Constituent variance processes}

Since its introduction, the univariate $\operatorname{GARCH}(1,1)$ model and its asymmetric generalization (TGARCH) have proved to be suitable for describing a wide variety of financial market data (Bollerslev 1986, Glosten et al. 1993, Bollerslev et al. 1994). We therefore employ this parsimonious specification of conditional heteroskedasticity in order to isolate the correlation dynamics. For each asset $i$, we presume a TGARCH(1,1) representation of the following form:

$$
\begin{aligned}
r_{i, t} & =\log \left(\frac{S_{i, t}}{S_{i, t-1}}\right)=\mu_{i, t}-\gamma_{i, t}+z_{i, t}, i=1, \ldots, N, \\
z_{i, t} & =e_{i, t} \sqrt{h_{i i, t}}, \quad e_{i, t} \sim(0,1), \\
h_{i i, t} & =\phi_{i, 0}+\phi_{i, 1} z_{i, t-1}^{2}+\phi_{i}^{-} z_{i, t-1}^{2} 1_{\left[z_{i, t-1}<0\right]}+\phi_{i, 2} h_{i i, t-1},
\end{aligned}
$$

where $1_{[\bullet]}$ denotes an indicator function, and $e_{i, t}$ is a univariate mean-zero, unit-variance innovation sequence characterized by cross sectional contemporaneous correlation. A leverage effect is captured by the TGARCH model in (6) if $\phi_{i}^{-}>0$. For notational convenience, asset specific TGARCH parameters are collected in $\phi_{i}=\left(\phi_{i, 0}, \phi_{i, 1}, \phi_{i}^{-}, \phi_{i, 2}\right)^{\top}$. As in Christoffersen et al. (2010) we add a compensator $\gamma_{i, t}$ to the mean equation (4) which ensures that the conditional expected gross rate of return equals the drift under the physical measure, i.e. we 
require $\mathrm{E}^{P}\left[S_{i, t} / S_{i, t-1} \mid \mathcal{F}_{t-1}\right]=\exp \left(\mu_{i, t}\right)$, which implies $\exp \left(\gamma_{i, t}\right)=\mathrm{E}^{P}\left[\exp \left(z_{i, t}\right) \mid \mathcal{F}_{t-1}\right]$

To model the asset specific risk premia $\mu_{i, t}$ we draw upon the conditional variant of the capital asset pricing model (CAPM), originally due to Sharpe (1964) and Lintner (1965). More precisely, we assume that

$$
\mu_{i, t}=r_{f, t}+\lambda \operatorname{Cov}_{t-1}\left[z_{i, t}, r_{m, t}\right], \quad i=1, \ldots, N
$$

where $r_{f, t}$ is the risk free rate and $r_{m, t}$ the excess return on the market portfolio, which we identify with the DAX index in our case. Since (7) holds also for the market, $\lambda$ has the interpretation of the market price of risk. Arguably it is restrictive to adopt a one-factor model and to assume a constant market price of risk, but we point out that this benchmark model is underlying many empirical studies (e.g. Giovannini and Jorion (1989) and Chan et al. (1992)) and can be confirmed empirically (de Santis and Gerard (1997)).

In setting out the TGARCH model, the conditional distribution in (5) is left unspecified. For the majority of its empirical applications to daily asset returns the assumption of conditional normality is made for the purposes of QML estimation of $\phi_{i}$, while diagnostic tests typically hint at remaining leptokurtosis of model innovations. As is evident from the specification of the copula multivariate GARCH model in (2), the $\varepsilon_{i, t}$ are weighted sums of dependent innovations $\varepsilon_{t}=\Sigma^{-1 / 2} \eta_{t}$. Consequently, to allow for conditional leptokurtosis of the marginal processes we presume that $\eta_{i, t}$ follow generalized error distributions (GED) with mean zero, unit variance and common shape parameter $\delta$. The GED coincides with the Gaussian distribution for $\delta=2$, see Nelson (1991) for further details on the GED. ${ }^{3}$

\subsection{Dynamic conditional correlations}

Multivariate GARCH models are widely adopted to describe second order comovements in multiple asset return systems, see Bauwens et al. (2006) for a review. As a subclass of multivariate GARCH models, DCC specifications have been introduced to cope with the 
curse of dimensionality in high dimensional systems (Bollerslev 1990, Engle 2002, Tse and Tsui 2002, Pelletier 2006). In analogy to univariate GARCH models, the DCC variants in Engle (2002) and Tse and Tsui (2002) formalize a response of current correlation matrices to recent news and past correlations, while in Pelletier (2006) time-varying correlation patterns are generated from a finite set of constant correlation matrices invoked by a Markov state process. Since the latter approach can be seen as compromising between a constant and a fully dynamic correlation model, we prefer to directly work with the fully dynamic model as introduced in Engle (2002). It involves non-linear estimation of only a few parameters, and in absence of news, it allows the correlation of the system to adjust slowly to its unconditional levels.

To formalize dynamic conditional correlation consider the following covariance decomposition

$$
H_{t}=D_{t} R_{t} D_{t}, D_{t}=\operatorname{diag}\left(\sqrt{h_{11, t}}, \ldots, \sqrt{h_{N N, t}}\right)
$$

In (8) the elements of $D_{t}$ are standard deviations collected from the univariate TGARCH models. The DCC $(1,1)$ model as introduced by Engle (2002) reads as

$$
R_{t}=Q_{t}^{*-1 / 2} Q_{t} Q_{t}^{*-1 / 2}, \quad Q_{t}=\bar{Q}\left(1-\alpha_{1}-\alpha_{2}\right)+\alpha_{1} e_{t-1} e_{t-1}^{\top}+\alpha_{2} Q_{t-1} .
$$

In (9), $Q_{t}^{*}=Q_{t} \odot I_{N}$, where $\odot$ denotes element-by-element multiplication, and $\bar{Q}$ is the unconditional second moment matrix of stacked univariate GARCH residuals $e_{t}=\left(e_{1, t}, \ldots, e_{N, t}\right)^{\top}$. News response and persistence of the conditional correlation matrix are governed by the parameters $\left(\alpha_{1}\right)$ and $\left(\alpha_{1}+\alpha_{2}\right)$, respectively. The elements of $R_{t}$ are of the form $r_{i j, t}=$ $\left(q_{i i, t} q_{j j, t}\right)^{-1 / 2} q_{i j, t}$. By the Cauchy-Schwartz inequality, $R_{t}$ is a correlation matrix such that its diagonal elements are unity $\left(r_{i i, t}=1, i=1, \ldots, N\right)$. If $\alpha_{1}=\alpha_{2}=0$, the model reduces to a constant conditional correlation model (Bollerslev 1990).

A notable extension of the $\operatorname{DCC}(1,1)$ model is the asymmetric DCC (ADCC) (Cappiello et al. 2006), which allows for leveraged correlations responding to negative market news. Within the ADCC model the right hand side of (9) is replaced by

$$
Q_{t}=\left(1-\alpha_{1}-\alpha_{2}\right) \bar{Q}-\alpha^{-\bar{N}}+\alpha_{1}\left(e_{t-1} e_{t-1}^{\top}\right)+\alpha^{-}\left(n_{t-1} n_{t-1}^{\top}\right)+\alpha_{2} Q_{t-1},
$$


where $n_{t}=e_{t} \odot \mathbf{1}_{\left[e_{t}<0\right]}$ and $\mathbf{1}_{\left[e_{t}<0\right]}$ is an $N$-dimensional vector of indicator functions with unit elements if $e_{i, t}<0 . \bar{N}$ is the unconditional second moment matrix of $n_{t}$. The dynamic correlation matrix is again determined as $R_{t}=Q_{t}^{*-1 / 2} Q_{t} Q_{t}^{*-1 / 2}$, which is positive definite with probability one if $\left(1-\alpha_{1}-\alpha_{2}\right) \bar{Q}-\alpha^{-} \bar{N}$ is positive definite (Cappiello et al. 2006). If $\alpha^{-}>0$, the ADCC model captures the common view that conditional correlations respond more strongly to bad news than to good news.

\subsection{Conditional dependence}

In most contributions to multivariate $\mathrm{GARCH}$ techniques, model innovations are presumed to be independent and identically (iid) Gaussian distributed over both the time and the cross sectional dimension. To address joint non Gaussian return distributions two main approaches can be distinguished. On the one hand, higher order unconditional cross moments have been considered to carry informational content for an assets' return distribution. In this framework the empirical co-skewness or co-kurtosis (e.g. of an asset with the market) enters equilibrium asset prices or portfolio allocations (see Chung et al. (2006) with further references). On the other hand, drawing upon Sklar's theorem, multivariate models have been developed which strictly separate the marginal distributional features of innovations from copula implied cross sectional dependence. In this framework generalizations towards time varying correlation are formalized by means of a structural model supposed to describe the copula parameter(s) in a predetermined or exogenous form (Granger et al. 2006, Patton 2006, Kim et al. 2008, Fantazzini 2009, Jin 2009, Creal et al. 2011, Christoffersen, Errunza, Jacobs and Langlois 2011). As such, although offering some enhanced generality of cross sectional dependence, the correlation patterns fitting in this model class are subject to strong cross sectional homogeneity restrictions. Lee and Long (2009) suggest a copula based multivariate GARCH setting, where time variation of second order moments and unconditional dependence of model innovations are conceptually separated but modeled simultaneously. To specify the former, they propose established multivariate GARCH specifications (e.g. DCC), 
while the latter is formalized by means of a parametric copula distribution function. Thus, similar to the motivation of DCC models which generalize the constant correlation case, this framework allows for a richer (dynamic) structure of cross asset volatility in comparison with the homogeneous correlation models. Accounting for these considerations, we adopt the model by Lee and Long (2009).

We implement the C-(A)DCC model in (3), (9) and (10) by means of the Clayton copula in light of economic and technical considerations. From the former perspective, the copula should be capable to characterize the index skew, which can be accomplished by choosing a copula having positive lower tail-dependence and zero upper tail-dependence. ${ }^{4}$ Thus, conditional on a large negative innovation hitting stock $i$, the probability that stock $j$ is also struck by a large negative innovation is bounded away from zero. Note, however, that vector innovations $\varepsilon_{t}$ are not directly drawn from the multivariate distribution, but are obtained as a standardized draw from the underlying copula. As an implication of this standardization, $\varepsilon_{t}$ and $\eta_{t}$ might differ with regard to their level of potential tail-dependence. In the following, we will however not distinguish between the tail-dependence characteristics of $\varepsilon_{t}$ and $\eta_{t}$ (in this regard see also Lee and Long (2009), p. 214, footnote 12).

¿From a technical perspective, numerical tractability of the copula density is essential to avoid prohibitive complexity of the log-likelihood evaluation. Noting that asymmetric taildependence could also be generated from other copula distributions (e.g. the (rotated) Gumbel), we adopt the Clayton copula, since an analytical expression of its density function is easy to obtain and the simulation of high-dimensional random vectors is straight forward. In Section 5.2 this choice is justified by means of a specification test following the lines of Genest and Rémillard (2008).

Formally, the Clayton copula reads as:

$$
C\left(u_{1}, \ldots, u_{N} ; \theta\right)=\left\{\left(\sum_{i=1}^{N} u_{i}^{-\theta}\right)-N+1\right\}^{-1 / \theta}, \quad \theta>0 .
$$

As $\theta \rightarrow 0$, the Clayton copula approaches the independence copula. The lower and upper 
tail-dependence are, respectively, $2^{-1 / \theta}$ and 0 . Similar to the models proposed in Granger et al. (2006) or Patton (2006), the copula multivariate GARCH framework could also allow for a time dependent parametrization of the copula parameter $\theta_{t}$. Since a main concern of our modeling approach is parsimony we renounce on such a refinement, see Lee and Long (2009) or Giacomini et al. (2006).

\section{Maximum likelihood estimation}

\subsection{The likelihood function and general considerations}

The log-likelihood function for the C-(A)DCC model is

$$
\begin{aligned}
\ell(\alpha, \phi, \theta, \delta, \lambda, \bar{Q}, \bar{N})= & \sum_{t=1}^{T} \log \left[f\left(r_{t} ; \alpha, \phi, \theta, \delta, \lambda, \bar{Q}, \bar{N}\right)\right] \\
= & \sum_{t=1}^{T} \sum_{i=1}^{N} \log \left[f_{i}\left(\eta_{i, t}\right)\right]+\log \left[c\left(F_{1}\left(\eta_{1, t}\right), \ldots, F_{N}\left(\eta_{N, t}\right)\right)\right] \\
& +\frac{T}{2} \log |\Sigma|-\frac{1}{2} \sum_{t=1}^{T} \log \left|H_{t}\right| .
\end{aligned}
$$

In $(12) c\left(u_{1}, \ldots, u_{N}\right)=\frac{\partial^{N} C\left(u_{1}, \ldots, u_{N}\right)}{\partial u_{1} \cdots \partial u_{N}}$ denotes the copula density function and the last two terms depend on the Jacobian of the transformation from observables in $r_{t}$ to the underlying innovations $\eta_{t}$.

Maximization of the likelihood of the C-(A)DCC-in-mean model is intricate, since first and second order data features have to be respected simultaneously. But even when proceeding from the simplifying assumption that the mean process in (1) is constant, one-step estimation, as originally suggested by Lee and Long (2009), is hardly feasible in systems with dimensions larger than three. Moreover, such an approach would sacrifice the separation of marginal volatility processes and correlation dynamics, which has been a principal argument for the feasibility of dynamic correlation modeling in high dimensional systems. On the other 
hand, the common three step estimation procedure due to Engle (2002) is known to suffer from an incidental parameter problem, which affects the news response parameters $\alpha_{1}$ and $\alpha^{-}$already in moderately sized set-ups of 30 to 50 assets to an economically relevant extent (Lancaster 2000, Engle and Sheppard 2001, Engle et al. 2009). It is therefore reasonable to expect - and empirical results reported in an earlier draft of this paper appear to confirm this interpretation - that also the copula based DCC model is afflicted with this short-coming.

For this reason, we still adopt a three-stage log-likelihood optimization, but follow recent suggestions of Engle et al. (2009) in implementing a composite maximum likelihood (CML) estimator. CML involves averaging the quasi-likelihoods of bivariate subsets of assets. Each of these subsets provides a valid quasi-likelihood, but is only weakly informative about parameters. As demonstrated in Engle et al. (2009) this approach can handle parameter estimation even in settings where the cross section of assets is larger than the time-series dimension, but does not suffer from the incidental parameter problem. In our case such CML estimation is simplified due to the homogeneity assumption imposed on the dependence structure, which is reflected in the single copula parameter $\theta$. This implies that the offdiagonal elements in $\Sigma$ are identical for all $i, j, i \neq j$ (diagonal elements are equal to unity for the purpose of identification).

\subsection{Estimation of the variance equations}

Initializing an outer loop of model estimation, we assume that the mean process in (1) is constant, i.e. $\mu=\mu_{t}-\gamma_{t}$, and work with the centered series of $r_{i, t}$. As proposed in de Santis and Gerard (1997) nontrivial conditional mean parameters can be recovered at later iteration steps based on second order filtered data. By standard QML techniques we obtain estimates of the marginal TGARCH volatility models for each asset specific return process $r_{i, t}, i=1, \ldots, N$.

In a second step, the unconditional matrices $\bar{Q}$ and $\bar{N}$ are estimated by means of the method 
of moment estimators ${ }^{5}$

$$
\widehat{\bar{Q}}=T^{-1} \sum_{t=1}^{T} \widehat{e}_{t} \widehat{e}_{t}^{\top}, \text { and } \widehat{\bar{N}}=T^{-1} \sum_{t=1}^{T} \widehat{n}_{t} \widehat{n}_{t}^{\top}
$$

In the third step, we jointly estimate the DCC parameter vector $\alpha=\left(\alpha_{1}, \alpha_{2}, \alpha^{-}\right)^{\top}$, the copula parameter $\theta$, and the shape parameter $\delta$ by means of CML techniques. To this end, we form subsystems $\left\{\widetilde{r}_{m, t}\right\}_{m=1}^{M}$, where each item $\widetilde{r}_{m, t}=\left(r_{i, t}, r_{j, t}\right)^{\top}, i \neq j$, is a bivariate vector of the returns of two distinct assets. More precisely, we use the set of contiguous returns which consists of $M=N-1=29$ pairs $\widetilde{r}_{1, t}=\left(r_{1, t}, r_{2, t}\right)^{\top}, \widetilde{r}_{2, t}=\left(r_{2, t}, r_{3 t}\right)^{\top}, \ldots, \widetilde{r}_{N-1, t}=$ $\left(r_{N-1, t}, r_{N t}\right)^{\top}$, where the numbering follows the alphabetical order as in Table $1 .{ }^{6}$ For given market price of risk $\lambda$ the composite log likelihood is given by

$$
\ell^{c}(\alpha, \theta, \delta \mid \phi, \bar{Q}, \bar{N})=\frac{1}{M} \sum_{t=1}^{T} \sum_{m=1}^{M} l_{m, t}\left(\alpha, \theta, \delta \mid \phi, \bar{Q}_{m}, \bar{N}_{m}\right) .
$$

where $l_{m, t}\left(\alpha, \theta, \delta \mid \phi, \bar{Q}_{m}, \bar{N}_{m}\right)=\log \left[f\left(\widetilde{r}_{m, t} ; \alpha, \theta, \delta\right)\right]$, while $\bar{Q}_{m}$ and $\bar{N}_{m}$ denote the relevant submatrices of $\bar{Q}$ and $\bar{N}$.

In each iteration during the maximization of (14), we compute $\Sigma$ numerically from of Hoeffding's lemma (Hoeffding 1940)

$$
\sigma_{i, j}(\theta)=\iint\left[F_{i j}\left(\eta_{i}, \eta_{j} \mid \theta\right)-F_{i}\left(\eta_{i}\right) F_{j}\left(\eta_{j}\right)\right] d \eta_{i} d \eta_{j}
$$

where $F_{i j}(\cdot)$ denotes a joint distribution function with corresponding marginals $F_{i}(\cdot)$ and $F_{j}(\cdot)$. In light of Sklar's Theorem (Sklar 1959), $F_{i j}\left(\eta_{i}, \eta_{j} \mid \theta\right)$ is replaced by the copula function $C\left(\eta_{i}, \eta_{j} \mid \theta\right)$. The double integral in (15) is evaluated in terms of Riemann sums. In each iteration, the likelihood is evaluated based on $\widehat{\eta}_{t}=\Sigma^{1 / 2} \widehat{\varepsilon}_{t}$, where $\widehat{\varepsilon}_{t}$ are the standardized residuals obtained from the first two steps.

\subsection{Estimation of the mean equations}

As a first step to the estimate the market price of risk in the mean equation (7), we compute the covariance $\widehat{\operatorname{Cov}}_{t-1}\left[r_{i, t}, r_{m, t}\right]$ from the estimated covariance system $\widehat{H}_{t}, t=1, \ldots, T$, as 
follows. Denote the index by $B_{t}=\sum_{i=1}^{N} a_{i} S_{i, t}$, which is given by the weighted sum of its constituents with absolute shares $a_{i}, i=1, \ldots, N$ (see Section 5.1 for a description of the DAX index shares). Moreover, denote by $w_{i}=a_{i} S_{i, t} / B_{t}$ the relative weight of asset $i$ in the index in period $t$. We then have

$$
\begin{aligned}
\operatorname{Cov}_{t}\left[r_{i, t+1}, r_{m, t+1}\right] & =\operatorname{Cov}_{t}\left[r_{i, t+1}, \sum_{j=1}^{N} w_{j} r_{j, t+1}\right] \\
& =\sum_{j=1}^{N} w_{j} \operatorname{Cov}_{t}\left[r_{i, t+1}, r_{j, t+1}\right] \\
& =\sum_{j=1}^{N} w_{j} h_{i j, t} .
\end{aligned}
$$

By replacing in (16) the population values by the elements of the estimated $\widehat{H}_{t}$, we obtain estimates $\widehat{\operatorname{Cov}}_{t}\left[r_{i, t}, r_{m, t}\right]$.

In a second step we run the regressions

$$
r_{i, t}-r_{f, t}=\lambda \widehat{\operatorname{Cov}}_{t-1}\left[r_{i, t}, r_{m, t}\right], \quad i=1, \ldots, N, \quad t=1, \ldots, T
$$

This is a classical regression in the asset pricing literature dating to French et al. (1987) and can be addressed by a number of panel regression techniques. We employ the mean group estimator, which amounts to averaging across $N$ separate OLS or weighted LS regression estimates. More precisely, the mean group estimator is given by $\widehat{\lambda}^{M G}=N^{-1} \sum_{i=1}^{N} \widehat{\lambda}_{i}$ and has variance $\operatorname{Var}\left[\widehat{\lambda}^{M G}\right]=[N(N-1)]^{-1} \sum_{i=1}^{N}\left(\widehat{\lambda}_{i}-\widehat{\lambda}^{M G}\right)^{2}$, see Pesaran and Smith (1995) for more details and further references.

Having accomplished these steps, we obtain estimates for the entire system. As mentioned, the procedure could be repeated based on a filtered return series. We will renounce on this second loop, since we barely find the market price of risk to be significant at single stock levels. It is only at the mean group level that we identify a significant positive market price of risk. We will discuss this issue in more detail in the empirical section. 


\section{Change of measure and estimation of option prices}

The success of GARCH type volatility specifications in time series econometrics has been followed by wide spread adoption of this model class for financial purposes, such as derivatives pricing (Duan 1995, Christoffersen and Jacobs 2004). The estimation of GARCH models is, however, accomplished under a physical measure $P$. For option pricing within the Harrison and Kreps (1979) framework, an equivalent martingale measure needs to be chosen. For instance, Duan (1995) introduced a GARCH based simulation approach for option valuation, in which the pricing parameters are identified from historical observations and risk neutralization is achieved by adjusting asset specific drift terms. Barone-Adesi et al. (2010) propose to directly calibrate observed prices by means of empirical risk neutral martingale sequences featuring GARCH type volatility. Given the number of parameters, a genuine calibration to market prices is not feasible in our case. We therefore apply a multivariate measure change of the GARCH process. In the following, we first present the theory that underlies the change of measure, and second, detail the implementation of the simulation based pricing algorithm.

\subsection{Change of measure}

Owing to the fundamental theorem of asset pricing (Dalang et al. 1990), arbitrage free option prices can be calculated as discounted expected payoffs if and only if the discounted price process of the underlying asset is a martingale under a so-called risk neutral probability measure. In the present setup, the price process of the index constituents under $P$ is given by

$$
S_{i, t}=S_{i, t-1} e^{\mu_{i, t}-\gamma_{i, t}+z_{i, t}}
$$

where $z_{i, t} \sim\left(0, h_{i i, t}\right)$ exhibits some zero-mean, heteroscedastic distribution. Clearly, these processes do not meet the martingale property, which requires a change to an equivalent martingale measure (EMM) denoted by $Q$. 
Our change of measure builds on the Esscher transform of the conditional physical probability measure and therefore is applicable under conditional heteroscedasticity and nonnormality. This use of the conditional Esscher transform is due to Gouriéroux and Monfort (2007) and Christoffersen et al. (2010), but the Esscher transform is a well-established technique in mathematical finance and insurance, see Esscher (1932), Gerber and Shiu (1994), Bühlmann et al. (1996), Kallsen and Shiryaev (2002) and the review article by Hubalek and Sgarra (2006), and is also used in Rombouts and Stentoft (2011). An overview of econometric modeling based on the conditional Esscher transform can be found in Bertholon et al. (2008).

For the construction of an EMM consider the following, $\mathcal{F}_{t}$-measurable and exponentially affine Radon-Nikodym process:

$$
\frac{d Q}{d P} \mid \mathcal{F}_{t} \equiv L_{t}=\exp \left\{-\left[\sum_{j=1}^{t} \nu_{j}^{\top} z_{j}+\Psi_{j}\left(\nu_{j}\right)\right]\right\},
$$

where $\Psi_{j}\left(\nu_{j}\right)$ is the natural logarithm of the conditional MGF of $z_{t}=\left(z_{1, t}, \ldots, z_{N, t}\right)^{\top}$, i.e. $\mathrm{E}^{P}\left[\exp \left(-u^{\top} z_{t}\right) \mid \mathcal{F}_{t-1}\right]=\exp \left(\Psi_{t}(u)\right)$ with $u \in \mathbb{R}^{N}$ and $\left\{\nu_{t}\right\}$ is a $N$-dimensional, predetermined, non-stochastic sequence which needs to be chosen in a suitable manner to obtain the martingale property. It is straightforward to see that (18) is indeed a Radon-Nikodym process, since $(i)$ we have $L_{t}>0$ by construction, and $(i i) \mathrm{E}^{P}\left[L_{t}\right]=1$ follows from a repeated application of the law of iterated expectations, see Lemma 1.1 of Christoffersen et al. (2010) for the details of the argument. The following result is due to Gouriéroux and Monfort (2007).

Proposition 4.1. The probability measure $Q$ induced by $L_{t}$ is an EMM if and only if

$$
\Psi_{t}\left(\nu_{t}-\mathbf{1}^{(i)}\right)-\Psi_{t}\left(\nu_{t}\right)+\mu_{i, t}-r_{f, t}-\gamma_{i, t}=0, \quad i=1, \ldots, N
$$

where $\mathbf{1}^{(i)}$ is a vector with all elements being zero except the $i$-th element which is unity. 
Proof: We need to show that $e^{-r_{f, t}} \mathrm{E}^{Q}\left[S_{i, t+1} \mid \mathcal{F}_{t}\right]=S_{i, t}$.

$$
\begin{aligned}
e^{-r_{f, t} \mathrm{E}^{Q}\left[S_{i, t+1} \mid \mathcal{F}_{t}\right]} & =e^{-r_{f, t}} \frac{1}{L_{t}} \mathrm{E}^{P}\left[L_{t+1} S_{i, t+1} \mid \mathcal{F}_{t}\right] \\
& =e^{-r_{f, t}} \mathrm{E}^{P}\left[e^{-\nu_{t}^{\top} z_{t}-\Psi_{t}\left(\nu_{t}\right)} S_{i, t} e^{\mu_{i, t}-\gamma_{i, t}+z_{i, t}} \mid \mathcal{F}_{t}\right] \\
& =S_{i, t} e^{\mu_{i, t}-r_{f, t}-\gamma_{i, t}-\Psi_{t}\left(\nu_{t}\right)} \mathrm{E}^{P}\left[e^{-\nu_{t}^{\top} z_{t}+z_{i, t}} \mid \mathcal{F}_{t}\right] \\
& =S_{i, t} \exp \left[\mu_{i, t}-r_{f, t}-\gamma_{i, t}-\Psi_{t}\left(\nu_{t}\right)+\Psi_{t}\left(\nu_{t}-\mathbf{1}^{(i)}\right)\right]
\end{aligned}
$$

where the first line follows from standard properties of Radon-Nikodym processes, see e.g. Lemma 5.2.2 in Shreve (2004), while the remaining lines follow from plugging in (17) and (18) and rearranging. Clearly the martingale property holds if and only if for all $i=1, \ldots, N$ condition (19) holds.

This approach yields a martingale measure for the filtration generated by all assets. In consequence the basket $B_{t}=\sum_{i=1}^{N} a_{i} S_{i, t}$ follows a martingale as well, since

$$
\begin{aligned}
e^{-r_{f, t}} \mathrm{E}^{Q}\left[B_{t+1} \mid \mathcal{F}_{t}\right] & =e^{-r_{f, t}} \mathrm{E}^{Q}\left[\sum_{i=1}^{N} a_{i} S_{i, t+1} \mid \mathcal{F}_{t}\right] \\
& =\sum_{i=1}^{N} a_{i} S_{i, t} \exp \left[\mu_{i, t}-r_{f, t}-\gamma_{i, t}-\Psi_{t}\left(\nu_{t}\right)+\Psi_{t}\left(\nu_{t}-\mathbf{1}^{(i)}\right)\right] \\
& =B_{t}
\end{aligned}
$$

by the very same line of arguments under condition (19).

Some remarks on the measure change are in order. Since the discrete time framework is an incomplete market setting, an infinite number of EMMs exist. The present approach picks but one out of this set. As has been shown in Christoffersen et al. (2010), conditional on using the Esscher transform, the choice is unique if the physical measure is infinitely divisible. Yet, it remains arbitrary in some sense, and other methods for finding an EMM could be used. Under certain conditions, however, the risk-neutral measure determined by means of the Esscher transform may coincide with those identified with other strategies, see Monoyios (2007) for further details. 


\subsection{Option pricing}

Option pricing requires a solution to (19). In certain cases this can be given exactly. Assume that $z_{t} \sim \mathcal{N}\left(0, H_{t}\right)$, i.e. $z_{t}$ follows a classical multivariate GARCH process. Then $\Psi_{t}(u)=$ $\frac{1}{2} u^{\top} H_{t} u$ and inserting into (19) yields, for $i=1, \ldots, N$,

$$
\begin{aligned}
0 & =\frac{1}{2}\left(\nu_{t}-\mathbf{1}^{(i)}\right)^{\top} H_{t}\left(\nu_{t}-\mathbf{1}^{(i)}\right)-\frac{1}{2} \nu_{t}^{\top} H_{t} \nu_{t}+\mu_{i, t}-r_{f, t}-\gamma_{i, t} \\
& =-\sum_{j=1}^{N} h_{i j, t} \nu_{j, t}+\frac{1}{2} h_{i i, t}-\gamma_{i, t}+\mu_{i, t}-r_{f, t} \\
& =-\sum_{j=1}^{N} h_{i j, t} \nu_{j, t}+\mu_{i, t}-r_{f, t}
\end{aligned}
$$

since $\frac{1}{2} h_{i i, t}=\gamma_{i, t}$ under normality. Putting this result into matrix notation and denoting by 1 a vector of ones yields

$$
H_{t} \nu_{t}=\mu_{t}-r_{f, t} \mathbf{1} \quad \Leftrightarrow \quad \nu_{t}=H_{t}^{-1}\left(\mu_{t}-r_{f, t} \mathbf{1}\right)
$$

which is the discrete time analogue to the solution of the market price of risk in the classical multidimensional security market based on the Geometric Brownian motion. The solution depends on the specification of the mean process $\mu_{t}$, only.

Unfortunately, for the copula multivariate GARCH framework the MGF of $z_{t}$ is not available. This poses three challenges. First, (19) does not admit an exact solution, second the RadonNikodym process is not explicit, and third we cannot construct and characterize the riskneutral distribution for option pricing. We address these three issues as follows.

1. As also suggested by Christoffersen et al. (2010), we determine an approximate solution of (19) by means of a second order Taylor expansion of $\Psi\left(\nu_{t}\right)$ and $\Psi\left(\nu_{t}-\mathbf{1}^{(i)}\right)$ in the neighborhood of zero. Noting that the gradient $\left.\nabla \Psi_{t}(-u)\right|_{u=0}=0$ and the $\left.\operatorname{Hessian}\left\{\Psi_{t}(-u)\right\}\right|_{u=0}=H_{t}$, this yields

$$
\nu_{t} \approx H_{t}^{-1}\left(\mu_{t}-r_{t} \mathbf{1}_{N}-\gamma_{t}+\frac{1}{2} h_{t}\right),
$$


where $\gamma_{t}=\left(\gamma_{1, t}, \ldots, \gamma_{N, t}\right)^{\top}$ and $h_{t}=\left(h_{11, t}, \ldots, h_{N N, t}\right)^{\top}$. In case of nonnormality the term $\frac{1}{2} h_{t}-\gamma_{t}$ drives a wedge between the risk-neutral and the physical distribution. Moreover, comparing (21) with (20) shows that the approximate and exact solution coincide in case of multivariate normality.

2. Similarly, a feasible Radon-Nikodym process is obtained by expanding $\Psi(u)$ in (18) to second order in the neighborhood of zero. This yields the approximate representation

$$
L_{t} \approx \widehat{L}_{t}=\exp \left\{-\left[\sum_{j=1}^{t} \nu_{j}^{\top} z_{j}+\frac{1}{2} \nu_{t}^{\top} H_{t} \nu_{t}\right]\right\} .
$$

As a consequence of the approximations (21) and (22) the effect of copula-specific higher order dependence vanishes. However, this is not without merit, as it allows us to interpret the copula shape parameter $\theta$ as a free parameter of the approximate Radon-Nikodym process $\widehat{L}_{t}=\widehat{L}_{t}(\theta)$. We hence receive an additional degree of freedom and can vary this parameter to study its impact on the implied volatility skew.

3. To overcome the lack of an explicit characterization of the risk neutral distribution we observe that such knowledge is not necessary, if the Radon-Nikodym process is known. Consider the valuation of a European style option with payoff function $g\left(S_{T}\right)=$ $\left(\xi\left(S_{T}-K\right)\right)^{+}$where $\xi=1$ for a call and $\xi=-1$ for a put and set $r_{f, t}=r_{f}$ for sake of simplicity. The option price is then given by

$$
V_{t}=e^{-r_{f} T} \mathrm{E}^{Q}\left[g\left(S_{T}\right) \mid \mathcal{F}_{0}\right]=e^{-r_{f} T} \mathrm{E}^{P}\left[L_{T} g\left(S_{T}\right) \mid \mathcal{F}_{0}\right]
$$

In consequence, simulating $S_{T}$ and $L_{T}$ under the measure $P$ gives rise to the following Monte Carlo estimator for the option price

$$
\begin{aligned}
V_{0} & =e^{-r_{f} T} \frac{1}{n} \sum_{i=1}^{n} \widehat{L}_{T}^{(i)}\left(\xi\left(S_{T}^{(i)}-K\right)\right)^{+} \\
& =e^{-r_{f} T} \frac{1}{n} \sum_{i=1}^{n}\left(\xi\left(\widehat{L}_{T}^{(i)} S_{T}^{(i)}-\widehat{L}_{T}^{(i)} K\right)\right)^{+}
\end{aligned}
$$

where $n$ denotes the number of simulation paths. 
Eq. (24) and (25) are equivalent, but it turns out that the latter representation is particularly useful. As is seen from (23), the discounted process $\left\{L_{t} S_{t}\right\}$ is a martingale under $P$; also $\left\{L_{t}\right\}$ is a martingale under $P$. To enhance the efficiency of the simulations, one can therefore apply the empirical martingale simulation scheme (EMS) proposed by Duan and Simonato (1998) to both $\left\{L_{t} S_{t}\right\}$ and $\left\{L_{t}\right\}$. The EMS serves as a variance reduction technique for the Monte Carlo estimate since it is a first order moment matching strategy. It consists of applying a multiplicative correction factor to simulated paths such that the simulated first moments coincide with their true means, see Duan and Simonato (1998) for the details. Sampling from (25) by means of the EMS scheme makes sure that $\left\{L_{t} S_{t}\right\}$ has exactly the forward price as mean and that the expectation of $\left\{L_{t}\right\}$ is unity. This ensures that the put-call parity holds, which is vital for the computation of implied volatility.

For basket option pricing, we simulate daily Monte Carlo TGARCH (log-)returns $r_{i, t}, i=$ $1, \ldots, 30$, in line with (5) and (6) and the (copula) (A)DCC dynamics according to (8), (9) and (10) using the CML/QML parameter estimates. Multivariate uniform variates with a dependence structure given by the Clayton copula are generated according to Algorithm 5.48 in McNeil et al. (2005). For drawing univariate GED random variables we follow Tadikamalla (1980). As drift we use a constant annual interest rate $r_{f}=2.61 \%$, which corresponds to the one-year EUR interbank offered rate on 7 December, 2005. The initial index level is $B_{0}=5266.75$. The simulation yields a 30-dimensional distribution of constituent asset prices $S_{i, T_{j}}$, at expiry dates $T_{j}, j=1, \ldots, 4$. At each expiry the value of the DAX is computed by $B_{T_{j}}=\sum_{i=1}^{30} a_{i} S_{i, T_{j}}$, where $a_{i}, i=1, \ldots, 30$, denote the shares of each stock in the index (see Table 1 for further details). Option prices are computed according to Eq. (25) with $B_{T}$ replacing $S_{T}$. For the Monte Carlo estimate we work with $n=10000$ paths, which we find to be a reasonable simulation size to attain a sufficient level of convergence. To avoid potential bias effects the seeds of the pseudo random number generators are set to the same value for each model. 
Finally, referring to (4) we delineate how to approximate the compensator $\gamma_{i, t}$. By definition $\gamma_{i, t}=\psi_{i, t}(1)$, where $\psi_{i, t}$ denotes the exponent of the marginal MGF of asset $i$ under $P$. Since this function is not available in closed form, it is determined by simulation. For the 30-dimensional system, we draw $m=50,000$ vectors $\eta$ from the Clayton copula using a candidate $\theta$ and the considered marginal distributions, and compute vector valued random variables $e=\widehat{\bar{Q}}^{1 / 2} \Sigma^{-1 / 2} \eta$. Hence, the elements in $e$ are correlated according to the unconditional correlation matrix of the system, but have unit variance. We now estimate the exponent of

the MGFs by means of the moment estimator given by $\widehat{\psi}_{i}^{e}(u)=\log \left[m^{-1} \sum_{j=1}^{m} \exp \left(-u e_{i j}\right)\right]$ for a dense grid of $u$ on some interval $\left[u_{l}, u_{u}\right]$. By the fact that $z_{i, t}=\sqrt{h_{i, t}} e_{i, t}$ and by the properties of the MGF we obtain as estimator of $\widehat{\gamma}_{i, t}=\widehat{\psi}_{i, t}(1)=\widehat{\psi}_{i}^{e}\left(\sqrt{h_{i, t}}\right)$ which can be evaluated at each time step. In order to reduce the computational burden, the moment estimator $\widehat{\psi}_{i}^{e}(u)$ is later replaced with a sixth order polynomial with even powers determined on $\left[u_{l}, u_{u}\right]$ by means of a least squares fit. As a matter of fact, the estimator $\widehat{\gamma}_{i, t}$ is an approximation in the sense that we miss the state-dependent correlation in each time step. However, taking state-dependent correlations into account appears infeasible. The procedure is repeated for each value of $\theta$ considered for option pricing.

\section{Empirical results}

\subsection{Data description}

For the empirical analysis we use historical index and stock price data and an associated cross section of index and single stock implied volatility data.

The historical price data cover the period from 5 February, 2001, to 7 December, 2005, and comprise the DAX, which is the major index of the German stock market, and all its 30 constituents $(T=1229)$. Stock prices are corrected for capital adjustments and dividend payments to avoid distortions in the estimation of the GARCH processes. Descriptive 
statistics are summarized in Table 1.

insert Table 1 about here

By definition, the DAX is a performance index, into which all dividends paid by its constituents are reinvested. Thus, to price derivatives with the DAX as underlying asset the dividend income stream must be set to zero. While this fact greatly simplifies derivatives valuation, a perfect reproduction of markets would require to set up a dividend model for all 30 stocks and, in the course of index path simulation, to make the corresponding adjustments to the index shares, when a dividend is paid by a constituent. To avoid this additional complexity we model the constituents without any dividend payments and hence do not adjust the index weights during the simulation. Since we are not interested in an exact replication of observed single stock option prices, but in the qualitative patterns of index and single stock skews the omission of dividends appears justified.

Throughout the analysis the basket (index) computation is done according to the weights given in the second last column of Table 1. These weights correspond to how the DAX index was calculated on 7 December, 2005. The weights change when a new stock is admitted to the index and exchanged against another one, when a corporate action takes place, or when - as discussed - a dividend is paid. ${ }^{7}$ As in the dividend case, we do not wish to model the admittance of new stocks or corporate actions. We therefore work with the implicit assumption that the index composition has not changed before 7 December, 2005, and will not change thereafter. For the time period under consideration this assumption is legitimate, since the last change took place on 22 September, 2003, the next following change took place on 19 December, $2005 .^{8}$ Thus, at least for almost two years of historical data the composition of the DAX index did not change. Starting on 7 December, 2005, we are left with a number of additional days to evaluate the out-of-sample pricing performance. We therefore take 7 December, 2005, as initial date for the present study. 
Option data consist of daily settlement implied volatility data of the DAX index and single stock options traded at the EUREX on 7 December, 2005. We consider four expiries, namely 72, 100, 191, 282 calendar days, summing up to 132 out-of-the money options. The DAX index option (ODAX) is of European style and belongs to the most liquidly traded index contracts in Europe. In contrast to index options, single stock options traded at the EUREX are of American style. A descriptive overview on the data is given in Table 2. As a means of filtering, we eliminate all options with an implied volatility larger than $80 \%$. It can be seen that the index implied volatility function has a more pronounced negative slope in comparison with individual equity option implied volatility functions. This is the stylized empirical fact we seek to capture in modeling the index by means of the multivariate setup. Note that the single stock implied volatility data are reported for illustrative purposes only and are not used in any of the estimations.

\section{$5.2 \quad$ In-sample estimation}

For space considerations we do not provide detailed estimation results for the univariate TGARCH processes characterizing the constituent return processes. Averaging the parameter estimates for the 30 DAX constituents we diagnose significant prevalence of leverage effects in volatility (see Table 3). Detailed results on estimated TGARCH volatility models for DAX constituents are available from the authors upon request.

insert Table 3 and Table 4 about here

Estimates for the correlation dynamics, the copula shape parameter and the GED shape parameter are displayed in Table 4. Parameter estimates for the correlation dynamics are very close to comparable results in the related literature (Engle and Sheppard 2001, Engle 
et al. 2009), but the standard errors for the news response parameters are likely to suffer from the limited time series information $(T=1229)$. Augmenting the correlation dynamics with a leverage parameter $\alpha^{-}$does not achieve a significant improvement of the fit as in the related literature (Longin and Solnik 2001, Ang and Chen 2002). Again this finding may be driven by the short time dimension of the data set. In the Gaussian model, the correlation response to negative news is small $\left(\hat{\alpha}_{1}+\hat{\alpha}^{-} \approx 0.035\right)$, but exceeds the news response quantified by means of the symmetric DCC model $\left(\hat{\alpha}_{1} \approx 0.022\right)$. To the largest extent correlation dynamics are driven by the autoregressive component $\left(\hat{\alpha}_{2} \approx 0.95\right)$. These findings are in line with other evidence of ADCC models, such as Cappiello et al. (2006).

With regard to tail characteristics of the marginal distributions it turns out that loglikelihood estimates are markedly in favor of the marginal GED specification, providing clear evidence of conditional leptokurtosis in DAX returns. The estimated GED shape parameter is $\widehat{\delta}=1.43$ for both the DCC and the ADCC model, which is far from the Gaussian distribution implied by $\delta=2$.

The estimation results for the C-(A)DCC models as documented in Table 4 show evidence for lower tail-dependence at a significance level of $10 \%$. Conditional on the marginal distribution, the copula parameter estimates are $\hat{\theta} \approx 0.07$ and $\hat{\theta} \approx 0.11$ under the Gaussian and GED marginals, respectively. These low parameter estimates are in line with the trivariate models considered in Lee and Long (2009) and are likely to reflect the strong homogeneity restriction imposed on the dependence structure over all constituents and over time. Intuitively, one would not expect too many events where the DAX constituents suffer simultaneously from a massive price deterioration. Noting that model diagnostics are markedly improved by the GED distribution, we also estimate (A)DCC models with leptokurtic marginals presuming an independence copula. A formal likelihood ratio test of the independence copula against the Clayton copula is significant at the $5 \%$ level for the DCC and ADCC models with GED marginals. This underscores the relevance of dependence beyond correlation. 
The better fit achieved by means of the Clayton copula does not fully justify this model choice, since other copula distributions might still outperform the Clayton copula based loglikelihood statistics. As an alternative way to assess if this specific model choice is supported by the data we subject it to a specification test proposed in Genest and Rémillard (2008). Comparing nine copula specification tests, Berg (2009) finds this test to be among the most preferable to detect deviations from a particular copula distribution supposed under the null hypothesis. The test builds on a Cramér-von Mises statistic contrasting the empirical copula and its parametric competitor, both evaluated by means of rank-based pseudo random variables. This allows to test the copula specification without making specific distributional assumptions on the margins. We compute the test statistic from residuals $\widehat{\eta}_{t}=\Sigma^{1 / 2} \widehat{\varepsilon}_{t}$, where $\Sigma=\Sigma(\widehat{\theta})$ is evaluated at the CML estimator $\widehat{\theta}$ (and $\widehat{\delta}$ for GED). ${ }^{9}$ Since the distribution of the test statistic is unknown, a bootstrap simulation needs to be implemented. The details of the bootstrap scheme are described in Appendix C.2 of Berg (2009). Based on $K=1000$ replications, the bootstrap $p$-values are 0.307 and 0.186 (0.062 and 0.089) for the DCC and ADCC model with Gaussian (GED) marginals, respectively. In conclusion, we cannot reject the Clayton copula assumption at the $5 \%$ significance level.

insert Table 5 about here

We estimate the market price of risk by means of OLS and WLS mean group estimators. For the latter the market variance is used to weight the observations, which can be computed from first stage estimates of $\widehat{H}_{t}$ analogously to (16). As documented in Table 5, WLS estimates turn out significant at the $5 \%$ level and close to unity for both the DCC and the ADCC model. Nevertheless, we caution about the interpretation of these results since the literature on fitting GARCH-in-mean models to daily returns typically tends to report statistically insignificant conditional mean parameters, see e.g. Bollerslev et al. (1994) and Blair et al. (2002). Only at lower frequencies, e.g. for monthly data, significant market prices of risk can be inferred, see Engle et al. (1987) and de Santis and Gerard (1997). We interpret this 
ambiguity as evidence for the existence of an economically meaningful market price of risk, which however cannot be statistically confirmed for single processes modeled at the daily frequency. For pricing options, we will therefore use - uniformly for all models and assets a market price of risk of $\lambda=1$.

\subsection{Model implied volatility skews}

Table 6 documents performance statistics for a couple of C-(A)DCC model specifications to assess their explanatory content for observed option prices. For convenience, the top row of Table 6 repeats the data of the implied volatility skews from Table 2. For model comparison we use two price based measures, namely the root mean squared (pricing) error (RMSE) and the mean absolute pricing error (MAPE) as in Barone-Adesi et al. (2010), and an implied volatility based RMSE. Implied volatility $\widehat{\sigma}$ is obtained by solving

$$
\widehat{\sigma}: \quad V_{0}-V_{0}^{B S}(\sigma)=0
$$

where $V_{0}^{B S}(\sigma)=\xi\left[S_{0} \Phi\left(\xi d_{1}\right)-e^{-r_{f} T} K \Phi\left(\xi d_{2}\right)\right]$ is the Black-Scholes valuation formula for calls $(\xi=1)$ and puts $(\xi=-1)$ with strike $K$ and expiry date $T$, with $\Phi$ denoting the cumulative density function of the Gaussian distribution, $d_{1}=\left[\log \left(S_{0} / K\right)+\left(r_{f}+\frac{1}{2} \sigma^{2}\right) T\right] /(\sigma \sqrt{T})$ and $d_{2}=d_{1}-\sigma \sqrt{T}$. The initial index or spot level is $S_{0}$, the risk-free rate $r_{f}$. In the right hand side panels of Table 6, we additionally document model implied skew characteristics at the index and average constituent level over the set of four maturities. As a measure of the implied volatility skew we use the symmetric difference $\widehat{\sigma}_{K=90 \%}-\widehat{\sigma}_{K=110 \%}$, where the percentage is relative to the initial index or spot level. For all simulation runs, the constants of the individual GARCH parameters, $\phi_{0}$, have been adjusted, such that the resulting term structure of basket implied volatility matches the observed term structure of DAX options. This adjustment is within the parameters' standard errors and serves to avoid a bias when comparing the performance statistics across alternative models.

As argued in the discussion of the approximate risk-neutralization, we interpret the copula 
shape parameter as a free variable. We therefore document pricing errors for three benchmark cases: $(i)$ the case of the independence copula, which is nested in the Clayton copula for $\theta \rightarrow 0$; $(i i)$ the CML estimator $\widehat{\theta}$ determined under $P$; (iii) a best fitting $\theta$ obtained by successively increasing $\theta$ in steps of 0.01 .

insert Table 6 about here

As is apparent from Table 6, for both types of modeling directions, i.e. DCC versus ADCC and Gaussian versus GED marginals, the cases of independence $(\theta=0)$ suffer from marked approximation losses relative to a scenario with mild tail-dependence as diagnosed under the physical measure $(\theta=\hat{\theta})$. All measures of fit are around $25 \%$ smaller than under the independence case. However, this fit can still be improved by allowing for slightly stronger tail-dependence. The best match is obtained for $\theta=0.33$, for which all measures of fit drop by an additional 30\%. As is visible from the right block of columns the improvement of the fit is due to substantially steeper basket implied volatility skews that emerge as a result of the higher cross sectional dependence.

insert Figure 1 and Figure 2 about here

Figures 1 and 2 contrast observed DAX option prices with model implied option prices as simulated from the best fitting C-ADCC model with GED innovations $(\delta=1.43)$ and $\theta=0.33$. Figure 1 shows market and model prices across a relative strike metric, the spot moneyness (strike divided by underlying price). As can be seen, out-of-the-money puts (spot moneyness less than one) tend to be underpriced across all expiries in comparison with out-of-the-money calls (spot moneyness larger than one) which in turn are too expensive, in particular for longer dated options. This impression is confirmed in Figure 2 displaying the corresponding implied volatility functions. The RMSE expressed in implied volatility is less than one percent (the exact figure is 0.779 , see Table 6 last row). Reading from 
the plot we observe that the mismatch can attain between one and two percentage points in volatility depending on the expiry. Despite these remaining discrepancies, we find the overall fit remarkably good when taking into consideration that it is obtained by variation of a single parameter and not by a direct calibration as in comparative studies (Engle and Mustafa (1992), Christoffersen and Jacobs (2004), Christoffersen et al. (2006), Barone-Adesi et al. (2010)).

Studying the model implied index skews, we observe that most models produce index skews which are conspicuously flatter than seen in the data. It is only the model implied volatility skew of the C-ADCC model (simulated with GED marginals and $\theta=0.33$ ) that matches the data for the shortest expiry; moving to longer time horizons, however, its index skew decays much faster than documented in Table 2. A similar observation applies when comparing index skews with average constituent skews. The symmetric DCC models either based on normal or GED marginals yield index skews that are never in excess of the average constituent skews, except in the case of highest cross sectional dependence. The asymmetric DCC models perform better, but only for the short-dated skews. For the long-term expiries at 191 and 282 days to maturity, the index skews drop to the levels measured for the average constituent skew.

insert Table 7 about here

The assessment of the C-ADCC model so far has been conditional on a particular trading day, namely 7 December, 2005. As a robustness check, we evaluate the models' out-of-sample pricing performance by simulating the C-ADCC model up to seven further trading days ahead. We use the parameter estimates as of 7 December, 2005, and only update the conditional covariance matrices given the realized shocks in the 30 underlying assets between 8 and 16 December, 2005. As documented in the data description, the DAX composition changed on 19 December, 2005. Therefore the out-of-sample evaluation period terminates on 16 December, 2005. The loss measures for the model based option prices and the model 
based implied volatilities are documented in Table 7 . Choosing the most preferable C-ADCC specification with copula parameter $\theta=0.33$ we find that all loss functions are of comparable magnitude as documented in Table 6. This observation also applies to the relative magnitude of basket versus constituent skews (not documented for sake of space). Hence the risk-neutralized physical process of speculative returns carries ex-ante predictive content for the stylized features of market implied volatility which is comparable to 'in-sample' model accuracy as measured on 7 December, 2005.

Overall, we conclude that the price processes estimated from time series data and riskneutralized by means of the Esscher transform fail to match the stylized features of index implied volatility skews in all respects. On the one hand this observation could indicate conceptual shortcomings of the considered exponentially linear pricing kernel. Evidence from the cross section of asset returns and from yield curve modeling suggests that quadratic (or otherwise nonlinear) pricing kernels, as studied e.g. in Dittmar (2002), Ahn et al. (2002), and Bakshi et al. (2010) and recently advanced in the discrete time framework by Christoffersen et al. (2011) and Monfort and Pegoraro (2011), might be empirically relevant. On the other hand, it is of interest to explore in depth the versatility of the present exponentially linear framework. Below we will follow this second route. We will ask: are there parameter constellations which better reproduce the stylized facts? And which insights do they provide for our understanding of flat constituent versus steep index skews? We address these issues by means of a sensitivity analysis.

\subsection{Sensitivity analysis}

Table 8 documents stylized features of simulated index and constituent option prices that are obtained by marginal variation of core parameters of the C-ADCC model generated from GED margins. First, the asymmetry parameters of the univariate TGARCH specifications $\left(\gamma_{i}^{-}\right)$are increased, while the ADCC parameters are kept constant. We choose parameters of 
$\bar{\phi}^{-}=0.089$ and $\bar{\phi}^{-}=0.098$, which imply, on average, a $10 \%$ and $20 \%$ increase of the leverage characterizing the estimated volatility models. Second, leaving the univariate second order dynamics unchanged, the leverage parameter of the correlation model is shifted upwards to $\alpha^{-}=0.030,0.051$ and $\alpha^{-}=0.101$ corresponding to a $20 \%, 200 \%$ and $400 \%$ increase in the leverage parameter. When varying leverage dynamics we simultaneously reduce the autoregressive parameters in the variance or the correlation equation such that the second order persistence remains unchanged in comparison with the estimated models. As a consequence the unconditional second order moments do not change under these variations and, hence, the term structure of implied volatility remains constant.

\section{insert Table 8 about here}

As can be seen in the top of Table 8 , increasing the parameter of the asymmetric second order dynamics at constituent levels acts positively on both the volatility skews of index options and the average constituent skew. In particular, if coupled with the cross sectional dependence prevailing at $\theta=0.33$, the small changes in the leverage variance parameter result in a basket skew close to the empirical figures in Table 2. Both the basket and the average constituent skews, are however similar in magnitude. Thus, asymmetries in the single stock variances cannot explain the empirically observed wedge between both skews. We find a similar comovement of index and constituent skews when reducing the GED shape parameter $\delta$ to allow for more heavy-tailedness in the conditional distributions (unreported results, available upon request).

Sensitivity results for the correlation response to bad news are displayed in the lower part of Table 8. For low cross sectional dependence, i.e. the C-ADCC model with $\theta=0$ (independence copula) and $\theta=0.11$ (CML estimate), the model implied skews of index and constituent volatilities are somewhat increased, but the average index skew is still of similar (or smaller) magnitude than the average constituent skew. This result holds irrespective of the level of the asymmetry parameter $\alpha^{-}$. Thus, even substantially leveraged corre- 
lation dynamics cannot explain the index skew under scenarios of independent or weakly tail-dependent vector innovations entering the dynamic system.

Setting $\theta=0.33$ and varying the correlation responses to bad news the C-ADCC model replicates the stylized features of implied volatility skews in a much better way. For the two choices $\alpha^{-}=0.051$ and $\alpha^{-}=0.101$ we find a marked excess index skew over the average constituent skew for short-dated expiries. But also for the longer maturities of 191 and 282 days, the simulation based index skew is well above the one quantified at the average constituent level. Nevertheless, despite these improvements, the model implied basket skew still decays at a faster rate over the various time horizons than is empirically documented in Table 2 .

As a final observation, inspecting the RMSE or MAPE loss measures for the two simulation designs $\theta=0.33, \alpha^{-}=0.051$ and $\theta=0.33, \alpha^{-}=0.101$ shows that a systematic calibration of model parameters to implied volatility would offer a closer fit to the actual implied volatility functions than can be achieved by means of the estimated model. For instance, the implied volatility RMSE statistic shrinks by around 35\% when replacing the CML estimates with $\alpha^{-}=0.101$ and $\alpha_{2}=.911$.

Summarizing the sensitivity analysis, we conclude that in the framework of the C-ADCC model the excess index skew cannot be driven by a leverage effect in the variance dynamics. Rather the principal sources are some level of lower tail-dependence coupled with leveraged correlation dynamics which are beyond the magnitudes estimated from stock return data. Steep index versus flat constituent skews do not emerge from either source alone. The intuition can be understood as follows. For pricing plain vanilla derivatives, it is not instantaneous correlation that matters but the terminal dependence structure at the relevant expiries. The terminal dependence structure among assets, however, does not change sufficiently when either of the features, i.e. cross sectional dependence or strongly leveraged correlation, appears alone. In this sense, leveraged correlation serves as a channel for cross 
sectional dependence to act on the terminal distribution such that steep index skews emerge.

\section{Conclusions}

We model the German DAX index, a major economy-wide stock basket comprising 30 blue chips, by means of the joint dependence of all constituent stocks in a multivariate model which features stochastic volatility, dynamic conditional correlation and conditional cross sectional dependence which is formalized by means of a copula as in Lee and Long (2009). Building on Engle et al. (2009), we adopt a CML/QML estimation strategy, which remains feasible in high dimensions, and suggest a theory for a change to an equivalent risk-neutral pricing measure following Gouriéroux and Monfort (2007) and Christoffersen et al. (2010). We simulate asset price paths along with an approximate Radon-Nikodym process under the physical measure to price European style index options. We then study the implied volatility skews which are generated by the model at the basket and at the constituents' level. Specifying constituent stocks as threshold GARCH processes, we allow for different types of dynamics, such as symmetric and asymmetric correlation dynamics, and impose alternative assumptions on the data generating process, such as Gaussian or fat-tailed innovations and zero versus positive (cross sectional) tail-dependence. Since all models are nested, the setup allows to disentangle the various sources responsible for the steepness of the implied volatility index skew.

We find that the implied volatility index skews obtained from the CML/QML estimates can explain a substantial fraction of the observed index skew, but still are too mild to match the empirical patterns. In an evaluation of out-of-sample pricing performance this result remains robust. For a deeper analysis, we vary selected parameters and study their impact on the skews. This sensitivity analysis demonstrates that cross sectional dependence coupled with a strong asymmetric news response in correlation generates a strong index and flat constituent skews. This conclusion holds irrespective of the distributional assumptions that underly the 
innovation process. We thus corroborate the common jump hypothesis of Branger and Schlag (2004), but additionally provide evidence for the role of asymmetric correlation dynamics which drive up the index skew relative to the average constituent skew.

Summarizing we find that the copula based ADCC model performs remarkably well in describing the index implied volatility surface as a basket of single stocks. To explain the remaining unexplained fraction of skewness in index implied volatility two main directions for future research arise. First, one could move beyond the exponentially affine pricing kernel and employ the more sophisticated specifications as suggested in Christoffersen et al. (2011) and Monfort and Pegoraro (2011). Second, given the importance of dependence beyond correlation, one could employ alternative copula models which strictly separate the marginal volatility processes and the copula, as suggested by Granger et al. (2006), Patton (2006), Kim et al. (2008), Fantazzini (2009), Jin (2009), Christoffersen, Errunza, Jacobs and Langlois (2011). It is likely that both directions provide additional insights on the relationship between single stock implied volatility and index skews. 


\section{Notes}

${ }^{1}$ Stochastic volatility, jumps and alternative distributional assumptions are well understood drivers of implied volatility in the univariate setting, see Hull and White (1987), Jorion (1988), Barndorff-Nielsen (1997), Bates (2000), Eraker et al. (2003) among many others. Formalized arguments for the relation between stochastic volatility and implied volatility are given in Renault and Touzi (1996) and Garcia and Renault (1998).

${ }^{2}$ In all instances we use the Cholesky decomposition as matrix square root for computational reasons. Since this choice might create an issue of lacking invariance with respect to variable ordering, we checked our results for robustness. We find that the estimates obtained by composite maximum likelihood do qualitatively not depend on variable ordering (see Section 3 for the details). The same applies to the simulation results of basket option prices and for the average implied volatility skews of constituent stocks. This is because our marginal variance processes are sufficiently homogeneous and cross-sectional dependence sufficiently moderate such that the total basket variance, which is the main driver of plain vanilla basket option prices, is hardly influenced by changing the order of names in the basket. All results for simulated basket option prices, which are reported in Section 5, are obtained for the order of appearance in Table 1. Clearly, an issue of invariance might arise if one were to consider pricing of strongly path-dependent options or best-of and worst-of options. Both topics are not within the scope of this work.

${ }^{3}$ Practically, it turns out that QML parameter estimates obtained for the variance processes are close to alternative estimates derived under more realistic innovation distributions (standardized $t$-distribution or GED). For this reason we estimate the univariate GARCH processes by means of QML, and later, treat the margins of the multivariate innovation vector $\eta_{t}=\Sigma^{1 / 2} \varepsilon_{t}$ to be leptokurtic as implied by the GED.

${ }^{4}$ For a formal definition of the concept of tail-dependence see e.g. McNeil et al. (2005), pp. 208-209.

${ }^{5}$ This step builds on the assumption that the unconditional expectations of $Q_{t}$ and of $e_{t-1} e_{t-1}^{\top}$ are identical. As argued in Aielli (2009) the factual violation of this assumption renders the estimators in (13) inconsistent. Aielli (2009) therefore proposes a consistent DCC model which is in full analogy to the model outlined in (1) to (3) except that the innovations $e_{t}$ in (9) are replaced by $\left(Q_{t} \odot I_{N}\right)^{1 / 2} e_{t}$. However, experience with our own simulations as well as the results reported in Aielli (2009) show that the actual bias is almost negligible in systems of bivariate dimension. Since CML estimation is built on bivariate subsets of assets, the empirical analysis relies upon the standard DCC model. 
${ }^{6}$ To check robustness of our choice of contiguous pairs, we also considered randomized pairs of returns with alternative dimensions of $M=29,99$. Over all these different choices CML estimates did not differ substantially. We therefore document the empirical results only for the contiguous pairs case.

${ }^{7}$ For a description of the terms and conditions of the DAX performance index, see Deutsche Börse (2007).

${ }^{8}$ On 22 September, 2003, Continental replaced MLP, and on 19 December, 2005 the Bayr. Hypo- und Vereinsbank was removed in favor of the Hypo Real Estate Holding. As an extraordinary event, on 31 January, 2005, Bayer AG split off part of its business (Lanxess AG).

${ }^{9}$ The assumptions on the margins enter via the second moment matrix $\Sigma$. The test statistic is, however, computed from the empirical margins obtained from the rank-based pseudo random numbers that are constructed from $\widehat{\eta}_{t}$. 


\section{References}

Ahn, D. H., R. F. Dittmar, and A. R. Gallant. 2002. Quadratic term structure models: theory and evidence. Review of Financial Studies 15: 243-288.

Aielli, G. P. 2009. Dynamic conditional correlations: on properties and estimation. Technical report, Department of Statistics, University of Florence.

Ang, A., and J. Chen. 2002. Asymmetric correlations of equity portofolios. Journal of Financial Economics 63: 443-494.

Bakshi, G., N. Kapadia, and D. Madan. 2003. Stock return characteristics, skew laws, and the differential pricing of individual equity options. Review of Financial Studies 16: 101143.

Bakshi, G., D. Madan, and G. Panayotov. 2010. Returns of claims on the upside and the viability of U-shaped pricing kernels. Journal of Financial Economics 97: 130-154.

Barndorff-Nielsen, O. E. 1997. Normal inverse Gaussian distributions and stochastic volatility modelling, Scandinavian Journal of Statistics 24: 1-13.

Barone-Adesi, G., R. F. Engle, and L. Mancini. 2010. Option valuation with conditional heteroskedasticity and nonnormality. Review of Financial Studies 23: 2139-2183.

Bauwens, L., S. Laurent, and J. V. K. Rombouts. 2006. Multivariate GARCH models: A survey. Journal of Applied Econometrics 31: 79-109.

Bates, D. S. 2000. Post-'87 crash fears in the S\&P 500 futures option market, Journal of Econometrics 94: 181-238.

Berg, D. 2009. Copula goodness-of-fit testing: an overview and power comparison. The European Journal of Finance 15: 675-701.

Bernard, C. and C. Czado. 2010. Multivariate option pricing using copulae. Technical report, University of Waterloo, Canada. 
Bertholon, H., A. Monfort, and F. Pegoraro. 2008. Econometric asset pricing modelling. Journal of Financial Econometrics 6: 407-458.

Black, F. and M. Scholes. 1973. The pricing of options and corporate liabilities. Journal of Political Economy 81: 637-654.

Blair, B., S. Poon, and S. Taylor. 2002. Asymmetric and crash effects in stock volatility for the S\&P100 index and its constituents. Applied Financial Economics 12: 319-329.

Bollen, N. and R. E. Whaley. 2004. Does net buying pressure affect the shape of the implied volatility functions? Journal of Finance 59 711-753.

Bollerslev, T. 1986. Generalized autoregressive conditional heteroscedasticity. Journal of Econometrics 31: 307-327.

Bollerslev, T. 1990. Modeling the coherence in short-run nominal exchange rates: a multivariate generalized ARCH model. Review of Economics and Statistics 72: 498-505.

Bollerslev, T., R. F. Engle, and D. B. Nelson. 1994. GARCH models, in R. F. Engle and M. D. L. (eds), Handbook of Econometrics, Elsevier, Amsterdam, 2961-3038.

Bollerslev, T., T. Law, and G. Tauchen. 2008. Risk, jumps, and diversification. Journal of Econometrics 144: 234-256.

Branger, N. and C. Schlag. 2004. Why is the index smile so steep? Review of Finance 8: $109-127$.

Bühlmann, H., F. Delbaen, P. Embrechts, and A. Shiryaev. 1996. No arbitrage, change of measure and conditional Esscher transform. CWI Quarterly 9: 291-317.

Cappiello, L., R. F. Engle, and K. Sheppard. 2006. Asymmetric dynamics in the correlations of global equity and bond returns. Journal of Financial Econometrics 4: 537-572.

Carr, P. and L. Wu. 2009. Variance risk premiums. Review of Financial Studies 22: 13111341. 
Chan, K., G. Karolyi, and R. Stulz. 1992. Global financial markets and the risk premium on U.S. equity. Journal of Financial Economics 32: 137-168.

Christoffersen, P., R. Elkamhi, B. Feunou, and K. Jacobs. 2010. Option valuation with conditional heteroskedasticity and nonnormality. Review of Financial Studies 23: 21392183.

Christoffersen, P., V. Errunza, K. Jacobs and H. Langlois. 2011. Is the potential for international diversification disappearing?, Working paper, McGill University, Montreal, Canada.

Christoffersen, P., S. Heston, and K. Jacobs. 2006. Option valuation with conditional skewness. Journal of Econometrics 131: 253-284.

Christoffersen, P., S. Heston, and K. Jacobs. 2011. A GARCH option model with variancedependent pricing kernel. AFA 2011 Denver Meetings Paper. Available at SSRN.

Christoffersen, P. and K. Jacobs. 2004. Which GARCH model for option valuation? Management Science 50: 1204-1221.

Chung, Y., H. Johnson, and M. Schill. 2006. Asset pricing when returns are nonnormal: Fama-French factors versus higher-order systematic co-moments. Journal of Business 79: 923-940.

Creal, D., S. J. Koopman and and A. Lucas. 2011. A dynamic multivariate heavy-tailed model for time-varying volatilities and correlations, Journal of Business 8 Economic Statistics . Forthcoming.

da Fonseca, J., M. Grasselli, and C. Tebaldi. 2008. A multifactor volatility Heston model. Quantitative Finance. Forthcoming.

Dalang, R. C., A. Morton, and W. Willinger. 1990. Equivalent martingale measures and no-arbitrage in stochastic securities market models. Stochastics 29: 185-201. 
de Santis, G. and B. Gerard. 1997. International asset pricing and portfolio diversification with time-varying risk. Journal of Finance 52: 1881-1912.

Deutsche Börse 2007. Guide to the Equity Indices of Deutsche Börse, 6.4 edn, Deutsche Börse AG, 60485 Frankfurt am Main, Germany.

Dittmar, R. F. 2002. Nonlinear pricing kernels, kurtosis preference, and evidence from the cross section of equity returns. Journal of Finance 57: 369-403.

Driessen, J., P. Maenhout, and G. Vilkov. 2009. The price of correlation risk: Evidence from equity options. Journal of Finance 64: 1377-1406.

Duan, J. C. 1995. The GARCH option pricing model. Mathematical Finance 5: 13-32.

Duan, J. C. and J. G. Simonato. 1998. Empirical martingale simulation for asset prices. Management Science 44: 1218-1233.

Elkamhi, R. and C. Ornthanalai. 2009. The market crash risk implicit in individual equities: Evidence from options and returns. Research paper, University of Iowa, Georgia Tech University.

Engle, R. F. 2002. Dynamical conditional correlation: A simple class of multivariate generalized autoregressive conditional heteroscedastic models. Journal of Business and Economic Statistics 20: 339-350.

Engle, R. F., D. M. Lilien, and P. R. Robbins. 1987. Estimating time varying risk premia in the term structure: the ARCH-M model. Econometrica 55: 391-407.

Engle, R. F., N. Shephard, and K. Sheppard. 2009. Fitting vast dimensional time-varying covariance models. Research paper series fin-08-009, NYU Stern School of Business.

Engle, R. F. and K. Sheppard. 2001. Theoretical and empirical properties of dynamic conditional correlation multivariate GARCH. Discussion paper 2001/15, Department of Economics, University of California, San Diego. 
Engle, R. and C. Mustafa. 1992. Implied ARCH models from options prices. Journal of Econometrics 52: 289-311.

Eraker, B., M. Johannes, and N. G. Polson. 2003. The impact of jumps in volatility and returns, Journal of Finance 58: 1269-1300.

Esscher, F. 1932. On the probability function in the collective theory of risk. Skandinavisk Aktuarietidskrift 15: 165-195.

Fantazzini, D. 2009. The effects of misspecified marginals and copulas on computing the value-at-risk: A monte carlo study. Computational Statistics and Data Analysis 53: 21682188.

French, K., G. Schwert, and R. Stambaugh. 1987. Expected stock returns and volatility. Journal of Financial Economics 19: 3-29.

Garcia, R. and E. Renault. 1998. A note on hedging in ARCH and stochastic volatility option pricing models. Mathematical Finance 8: 153-161.

Genest, C. and B. Rémillard. 2008. Validity of the parametric bootstrap for goodness-of-fit testing in semiparametric models. Annales de L'Institut Henri Poincaré - Probabilités et Statististiques 44: 1096-1127.

Gerber, H. and E. Shiu. 1994. Option pricing by Esscher transforms. Transactions of the Society of Actuaries 46: 98-140.

Giacomini, E., W. Härdle, E. Ignatieva, and V. Spokoiny. 2006. Inhomogenous dependency modelling with time varying copulae. Discussion paper, SFB 649, Humboldt-Universität zu Berlin.

Giovannini, A. and P. Jorion. 1989. The time variation of risk and return in the foreign exchange and stock markets. Journal of Finance 44: 307-325. 
Glosten, L., R. Jagannathan, and D. Runkle. 1993. Relationship between the expected value and the volatility of the nominal excess return on stocks. Journal of Finance 48: 1779-1801.

Goorbergh, R. v. d., C. Genest, and B. Werker. 2005. Bivariate option pricing using dynamic copula models. Insurance: Mathematics and Economics 37: 101-114.

Gouriéroux, C. and A. Monfort. 2007. Econometric specification of stochastic discount factor models. Journal of Econometrics 136: 509-530.

Gouriéroux, C. and R. Sufana. 2004. Derivative pricing with multivariate stochastic volatility: Application to credit risk. Working paper, University of Toronto.

Granger, C., T. Teräsvirta, and A. Patton. 2006. Common factors in conditional distributions for bivariate time series. Journal of Econometrics 132: 43-57.

Harrison, J. and D. Kreps. 1979. Martingales and arbitrage in multiperiod securities markets. Journal of Economic Theory 20: 381-408.

Hoeffding, W. 1940. Massstabinvariante Korrelationstheorie. Schriften des Mathematischen Seminars und des Instituts für angewandte Mathematik der Universität Berlin 5: 181-233.

Hubalek, F. and C. Sgarra. 2006. Esscher transforms and the minimal entropy martingale measure for exponential Lévy models. Quantitative Finance 6: 125-145.

Hull, J. and A. White. 1987. The pricing of options on assets with stochastic volatilities, Journal of Finance 42: 281-300.

Jin, X. 2009. Large portfolio risk management with dynamic copulas. Technical report, McGill University.

Jorion, P. 1988. On jump processes in the foreign exchange and stock markets, Review of Financial Studies 1: 427-445.

Kallsen, J. and A. Shiryaev. 2002. The cumulant process and Esschers change of measure. Finance and Stochastics 6: 397-428. 
Kim, G., M. Silvapulle, and P. Silvapulle. 2008. Estimating the error distribution in multivariate heteroscedastic time series models. Journal of Statistical Planning and Inference 138: $1442-1458$.

Lancaster, T. 2000. The incidental parameter problem since 1948. Journal of Econometrics 95: 391-413.

Langnau, A. 2010. A dynamic model for correlation. RISK 23: 74-78.

Lee, T. and X. Long. 2009. Copula-based multivariate GARCH model with uncorrelated dependent errors. Journal of Econometrics 150: 207-218.

Lintner, J. 1965. The valuation of risky assets and the selection of risky investments in stock portfolios and capital budgets. Review of Economics and Statistics 47: 13-37.

Longin, F. and B. Solnik. 2001. Extreme correlations of international equity markets. Journal of Finance 61: 649-676.

Luciano, E. and W. Schoutens. 2006. A multivariate jump-driven financial asset model. Quantitative Finance 6: 385-402.

McNeil, A., Frey, R. and P. Embrechts. 2005. Quantitative Risk Management: concepts, techniques, and tools. Princeton University Press, New York.

Mo, H. and L. Wu. 2007. International capital asset pricing: Evidence from options. Journal of Empirical Finance 14: 465-498.

Monfort, A. and F. Pegoraro. 2011. Asset pricing with second-order Esscher transforms. Research paper, CREST, Paris.

Monoyios, M. 2007. The minimal entropy measure and an Esscher transform in an incomplete market model. Statistics 85 Probability Letters 77: 1070-1076.

Nelson, D. B. 1991. Conditional heteroskedasticity in asset returns: A new approach. Econometrica 59: 347-370. 
Patton, A. 2006. Modeling asymmetric exchange rate dependence. International Economic Review 47: 527-556.

Pelletier, D. 2006. Regime switching for dynamic correlations. Journal of Econometrics 131: 445473 .

Pesaran, M. and R. Smith. 1995. Estimating long-run relationships from dynamic heterogeneous panels. Journal of Econometrics 68: 79-113.

Renault, E. and N. Touzi. 1996. Option hedging and implied volatilities in a stochastic volatility model. Mathematical Finance 6: 279-302.

Rombouts, J. and L. Stentoft. 2011. Multivariate option pricing with time varying volatility and correlations. Journal of Banking \& Finance 35: 2267-2281.

Rubinstein, M. 1994. Implied binomial trees. Journal of Finance 49: 771-818.

Şerban, M., J. Lehoczky, and D. Seppi. 2008. Cross-sectional stock option pricing and factor models of returns. Research paper, Morgan Stanley, Carnegie Mellon University.

Sharpe, W. 1964. Capital asset prices: A theory of market equilibrium under conditions of risk. Journal of Finance 19: 425-442.

Shreve, S. 2004. Stochastic calculus for finance II. Springer-Verlag, Berlin, Heidelberg.

Sklar, A. 1959. Fonctions de répartition à $n$ dimensions et leurs marges. Publications de l'Institut de Statistique de l'Université de Paris 8: 229-231.

Tadikamalla, P. R. 1980. On simulating non-normal distributions. Psychometrika 45: 273279.

Tse, Y. and A. Tsui. 2002. A multivariate generalized autoregressive conditional heteroscedastic model with time-varying correlations. Journal of Business and Economic Statistics 20: 351-362. 


\begin{tabular}{|c|c|c|c|c|c|c|c|c|c|}
\hline Constituent & Min & Max & Mean & Med & Std & Skew. & Kurt. & $\overline{\overline{\breve{a}_{i}}}$ & $\overline{w_{i}}$ \\
\hline DAX & -8.87 & 7.55 & -.019 & .033 & 1.76 & -0.05 & 5.54 & - & \\
\hline Adidas-Salomon & -11.53 & 8.63 & .068 & .041 & 1.85 & -0.14 & 7.13 & 2.56 & 1.23 \\
\hline Altana & -19.03 & 14.16 & .013 & -.021 & 2.26 & -0.56 & 12.81 & 3.92 & 0.57 \\
\hline Allianz & -15.14 & 12.66 & -.071 & .033 & 2.70 & -0.12 & 6.50 & 22.13 & 8.81 \\
\hline BASF & -8.25 & 13.70 & .035 & .022 & 1.87 & 0.51 & 8.45 & 29.29 & 5.81 \\
\hline Bayer & -18.97 & 33.01 & -.021 & -.046 & 2.55 & 1.21 & 29.97 & 40.82 & 4.38 \\
\hline BMW & -11.42 & 8.30 & .006 & .027 & 2.05 & -0.18 & 5.92 & 18.57 & 2.19 \\
\hline Commerzbank & -13.30 & 15.44 & -.008 & .000 & 2.55 & 0.13 & 7.95 & 30.44 & 2.44 \\
\hline Continental & -11.15 & 9.08 & .122 & .071 & 2.05 & 0.20 & 5.49 & 8.13 & 1.89 \\
\hline Deutsche Börse & -10.76 & 8.50 & .073 & .106 & 1.72 & -0.24 & 5.96 & 5.92 & 1.63 \\
\hline Deutsche Bank & -14.06 & 12.58 & -.002 & .041 & 2.32 & -0.15 & 6.42 & 28.91 & 7.67 \\
\hline Daimler Chrysler & -10.96 & 9.81 & .004 & -.027 & 2.23 & 0.00 & 5.09 & 48.62 & 6.59 \\
\hline Dt. Post & -9.33 & 8.63 & -.009 & .000 & 1.95 & -0.08 & 5.28 & 34.65 & 2.08 \\
\hline Dt. Telekom & -16.44 & 13.54 & -.065 & -.065 & 2.66 & 0.06 & 6.59 & 147.80 & 6.55 \\
\hline E.ON & -7.98 & 8.95 & .039 & .055 & 1.79 & 0.12 & 6.05 & 38.67 & 9.89 \\
\hline Fresenius Medical & -12.66 & 26.51 & .000 & -.014 & 2.26 & 1.08 & 20.55 & 1.92 & 0.49 \\
\hline Henkel KGaA & -7.71 & 7.11 & .019 & .029 & 1.51 & -0.07 & 6.32 & 3.32 & 0.88 \\
\hline Hypo-Vereinsbank & -15.67 & 14.14 & -.063 & -.068 & 2.95 & -0.12 & 6.13 & 33.57 & 2.66 \\
\hline Infineon & -15.44 & 16.06 & -.140 & -.129 & 3.64 & 0.02 & 5.00 & 34.16 & 0.84 \\
\hline Dt. Lufthansa & -16.36 & 15.71 & -.050 & -.096 & 2.42 & 0.06 & 9.13 & 23.39 & 0.87 \\
\hline Linde & -14.33 & 10.65 & .021 & .034 & 1.77 & -0.25 & 8.67 & 4.53 & 0.88 \\
\hline MAN & -11.39 & 11.08 & .034 & .028 & 2.37 & -0.08 & 5.54 & 7.88 & 1.08 \\
\hline Metro & -10.38 & 17.62 & -.016 & -.052 & 2.21 & 0.42 & 8.61 & 8.05 & 0.96 \\
\hline Münchener Rück & -17.04 & 13.23 & -.082 & -.036 & 2.65 & -0.33 & 8.02 & 10.29 & 3.73 \\
\hline RWE & -7.66 & 9.07 & .039 & .024 & 1.79 & 0.15 & 5.40 & 26.00 & 4.83 \\
\hline SAP & -15.11 & 22.67 & .003 & .000 & 3.00 & 0.76 & 9.89 & 11.89 & 5.79 \\
\hline Schering & -16.10 & 8.49 & .004 & .051 & 1.90 & -0.81 & 10.88 & 9.69 & 1.70 \\
\hline Siemens & -8.95 & 10.59 & -.026 & -.016 & 2.47 & 0.16 & 4.55 & 46.76 & 9.80 \\
\hline Thyssen Krupp & -12.04 & 7.70 & .005 & .063 & 2.12 & -0.11 & 5.30 & 23.00 & 1.25 \\
\hline Tui & -18.45 & 14.46 & -.053 & -.096 & 2.63 & -0.01 & 8.08 & 12.57 & 0.69 \\
\hline Volkswagen & -9.66 & 10.69 & -.002 & -.084 & 2.20 & -0.06 & 5.17 & 12.32 & 1.81 \\
\hline
\end{tabular}

Table 1: Descriptive summary statistics for constituent log returns from 6 February, 2001 until 7 December, 2005 ( $T=1229$ observations). Index weights (number of shares) $a_{i}$ for the DAX constituents are obtained after multiplying the weights given in the column $\breve{a}_{i}$ with the constant catenation factor $1000 / 6019.57$, i.e. $a_{i}=1000 \breve{a}_{i} / 6019.57$. Relative weights in column $w_{i}$ (in percentage units) are computed as of 7 December, 2005. 


\begin{tabular}{l|r|r|r|r||r|r|r|r}
\hline \hline & \multicolumn{4}{|c||}{ Index } & \multicolumn{4}{c}{ Constituents } \\
\hline Maturity (days) & \multicolumn{1}{|c|}{72} & \multicolumn{1}{|c|}{100} & \multicolumn{1}{c|}{191} & \multicolumn{1}{c|}{282} & \multicolumn{1}{c}{72} & \multicolumn{1}{c}{100} & \multicolumn{1}{c}{191} & \multicolumn{1}{c}{282} \\
\hline Min & 12.91 & 13.32 & 14.08 & 14.69 & 20.74 & 21.25 & 21.54 & 21.70 \\
Max & 18.60 & 18.81 & 18.91 & 19.23 & 23.15 & 23.41 & 23.38 & 23.56 \\
Mean & 15.10 & 15.54 & 16.29 & 16.79 & 21.48 & 21.99 & 22.27 & 22.47 \\
Median & 14.73 & 15.27 & 16.19 & 16.74 & 21.18 & 21.79 & 22.17 & 22.39 \\
Std & 1.88 & 1.82 & 1.55 & 1.42 & 0.77 & 0.69 & 0.60 & 0.61 \\
\hline Imp. vol. skew (\%) & 5.69 & 5.62 & 5.21 & 4.86 & 2.09 & 1.72 & 1.29 & 1.25 \\
\hline \hline
\end{tabular}

Table 2: Summary statistics for implied volatilities of the DAX and its constituents (averaged values) observed as of 7 December, 2005. Maturities are given in calender days. The implied volatility skew is defined as $\widehat{\sigma}_{K=90 \%}-\widehat{\sigma}_{K=110 \%}$, where $\widehat{\sigma}_{K}$ is the implied volatility of an option with strike $K$. 


\begin{tabular}{cccc}
\hline \hline $\bar{\phi}_{0}$ & $\bar{\phi}_{1}$ & $\bar{\phi}^{-}$ & $\bar{\phi}_{2}$ \\
\hline $6.3 \times 10^{-6}$ & 0.032 & 0.081 & 0.913 \\
$\left(4.0 \times 10^{-6}\right)$ & $\left(2.4 \times 10^{-2}\right)$ & $\left(3.1 \times 10^{-2}\right)$ & $\left(3.5 \times 10^{-2}\right)$ \\
\hline \hline
\end{tabular}

Table 3: Averaged QML TGARCH estimates and their average (robust) standard errors for the 30 DAX constituent return processes, i.e. excluding the DAX index, as listed in Table 1. Single stock variance equation is given by $h_{i i, t}=\phi_{i, 0}+\phi_{i, 1} z_{i, t-1}^{2}+\phi_{i}^{-} z_{i, t-1}^{2} 1_{\left[z_{i, t-1}<0\right]}+\phi_{i, 2} h_{i i, t-1}$, for $i=1, \ldots, 30$, where $z_{i, t}=e_{i, t} \sqrt{h_{i i, t-1}}$ and $e_{i, t} \sim \mathcal{N}(0,1)$ for the purpose of QML estimation. 


\begin{tabular}{|c|c|c|c|c|c|c|}
\hline & \multicolumn{5}{|c|}{ CML estimates } & Log-lik \\
\hline Model & $\widehat{\alpha}_{1}$ & $\widehat{\alpha}_{2}$ & $\widehat{\alpha}^{-}$ & $\widehat{\theta}$ & $\widehat{\delta}$ & \\
\hline DCC & $\begin{array}{c}0.0218 \\
(1.943)\end{array}$ & $\begin{array}{l}0.940 \\
(24.46)\end{array}$ & & & 2 & 6409.2 \\
\hline $\mathrm{ADCC}$ & $\begin{array}{c}0.0100 \\
(0.777)\end{array}$ & $\begin{array}{l}0.949 \\
(24.45)\end{array}$ & $\begin{array}{l}0.025 \\
(1.176)\end{array}$ & & 2 & 6410.0 \\
\hline DCC & $\begin{array}{c}0.0205 \\
(1.825)\end{array}$ & $\begin{array}{l}0.943 \\
(24.63)\end{array}$ & & & $\begin{array}{c}1.43 \\
(26.91)\end{array}$ & 6446.5 \\
\hline $\mathrm{ADCC}$ & $\begin{array}{c}0.0091 \\
(0.714) \\
\end{array}$ & $\begin{array}{l}0.951 \\
(25.01) \\
\end{array}$ & $\begin{array}{c}0.0250 \\
(1.127)\end{array}$ & & $\begin{array}{c}1.43 \\
(26.31) \\
\end{array}$ & 6447.2 \\
\hline $\mathrm{C}-\mathrm{DCC}$ & $\begin{array}{c}0.0212 \\
(1.904)\end{array}$ & $\begin{array}{l}0.943 \\
(25.25)\end{array}$ & & $\begin{array}{c}0.0675 \\
(1.607)\end{array}$ & 2 & 6410.8 \\
\hline $\mathrm{C}-\mathrm{ADCC}$ & $\begin{array}{c}0.0100 \\
(0.816)\end{array}$ & $\begin{array}{l}0.951 \\
(27.72) \\
\end{array}$ & $\begin{array}{l}0.024 \\
(1.118) \\
\end{array}$ & $\begin{array}{c}0.0661 \\
(1.485)\end{array}$ & 2 & 6411.4 \\
\hline C-DCC & $\begin{array}{c}0.0202 \\
(1.748)\end{array}$ & $\begin{array}{l}0.946 \\
(25.95)\end{array}$ & & $\begin{array}{c}0.1134 \\
(1.713)\end{array}$ & $\begin{array}{c}1.43 \\
(25.80)\end{array}$ & 6448.7 \\
\hline $\mathrm{C}-\mathrm{ADCC}$ & $\begin{array}{c}0.0094 \\
(0.733)\end{array}$ & $\begin{array}{l}0.953 \\
(25.97)\end{array}$ & $\begin{array}{l}0.023 \\
(1.029) \\
\end{array}$ & $\begin{array}{c}0.1108 \\
(1.787)\end{array}$ & $\begin{array}{c}1.43 \\
(25.31)\end{array}$ & 6449.3 \\
\hline
\end{tabular}

Table 4: Estimation results for the parameters governing the correlation dynamics $\left(\alpha_{1}, \alpha^{-}, \alpha_{2}\right)^{\top}$, the shape parameter $\delta$ of the marginal distribution of $\eta_{i, t}$ and the Clayton copula shape parameter $\theta$ for all considered C-(A)DCC-GARCH models $(t$-ratios given in parentheses). Correlation dynamics follow $Q_{t}=\left(1-\alpha_{1}-\alpha_{2}\right) \bar{Q}-\alpha^{-} \bar{N}+\alpha_{1}\left(e_{t-1} e_{t-1}^{\top}\right)+$ $\alpha^{-}\left(n_{t-1} n_{t-1}^{\top}\right)+\alpha_{2} Q_{t-1}$. The assumption of Gaussian innovations corresponds to $\delta=2$. The rightmost column block gives composite log-likelihood values. Sample period is from 6 February, 2001, until 7 December, 2005 ( $T=1229$ observations). 


\begin{tabular}{c|cc}
\hline \hline & DCC & ADCC \\
\hline OLS & 1.050 & 1.020 \\
& $(2.945)$ & $(2.867)$ \\
\hline WLS & 1.188 & 1.167 \\
& $(2.045)$ & $(2.085)$ \\
\hline \hline
\end{tabular}

Table 5: Market price of risk $\widehat{\lambda}$ for (A)DCC models estimated by means of a mean group estimator. WLS weights are estimated index variances obtained from first stage QML estimation. The sample period is from 6 February, 2001, until 7 December, $2005(T=1229$ observations). $t$-ratios given in parentheses. 


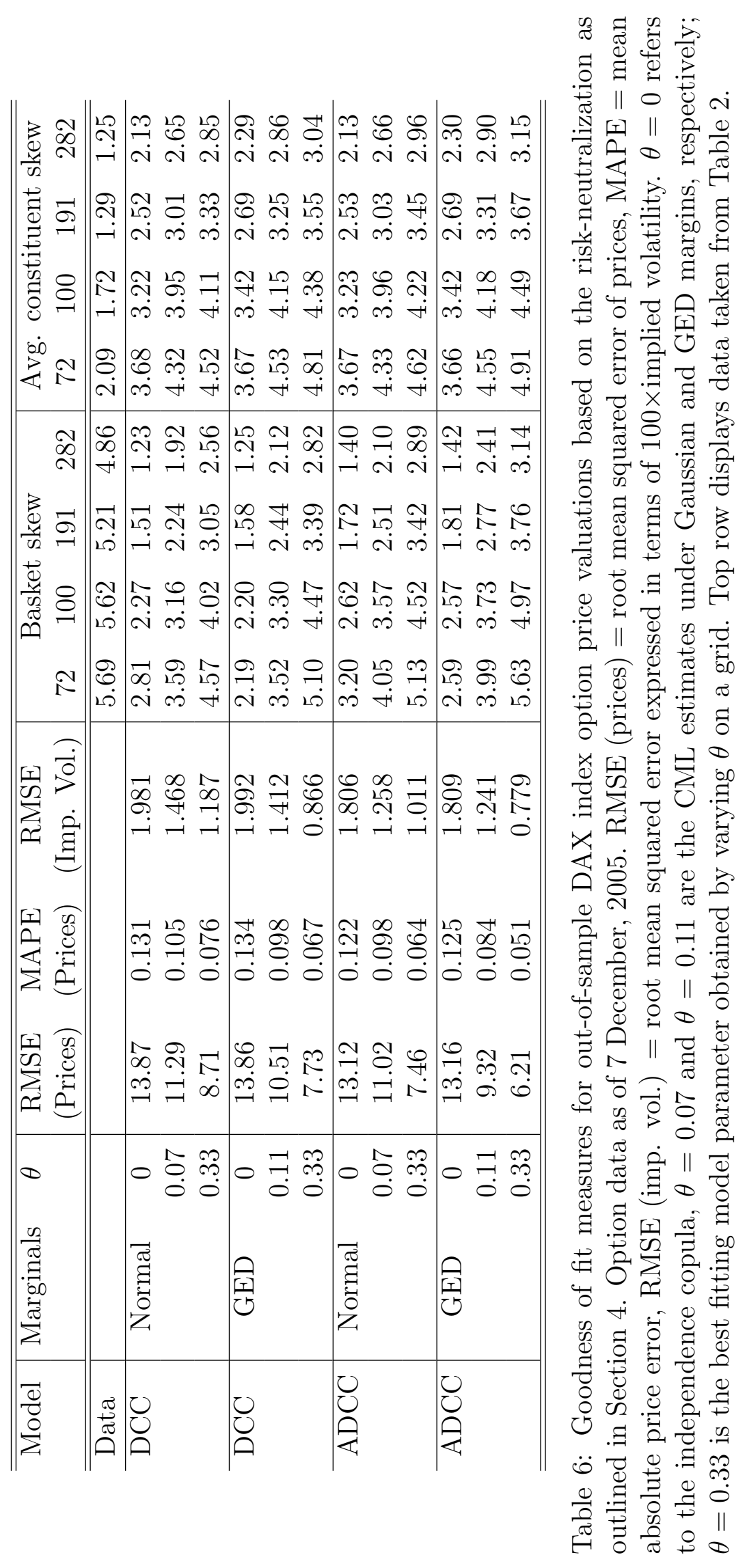




\begin{tabular}{c|ccc}
\hline \hline trading date & RMSE (prices) & MAPE (prices) & RMSE (imp. vol) \\
\hline $07 / 12 / 2005$ & 6.21 & 0.051 & 0.779 \\
$08 / 12 / 2005$ & 6.91 & 0.056 & 0.847 \\
$09 / 12 / 2005$ & 5.72 & 0.050 & 0.604 \\
$12 / 12 / 2005$ & 5.93 & 0.053 & 0.752 \\
$13 / 12 / 2005$ & 7.52 & 0.061 & 0.963 \\
$14 / 12 / 2005$ & 6.33 & 0.048 & 0.736 \\
$15 / 12 / 2005$ & 8.01 & 0.070 & 1.100 \\
$16 / 12 / 2005$ & 7.00 & 0.075 & 1.000 \\
\hline \hline
\end{tabular}

Table 7: Out-of-sample pricing for days following 7 December, 2005 based on the C-ADCC model with GED margins and $\theta=0.33$. All model parameters are kept constant as given in Table 4, while variances and correlations are daily updated according to the realized innovations. RMSE (prices) = root mean squared error of prices, MAPE = mean absolute price error, RMSE (imp. vol.) = root mean squared error expressed in terms of $100 \times$ implied volatility. 


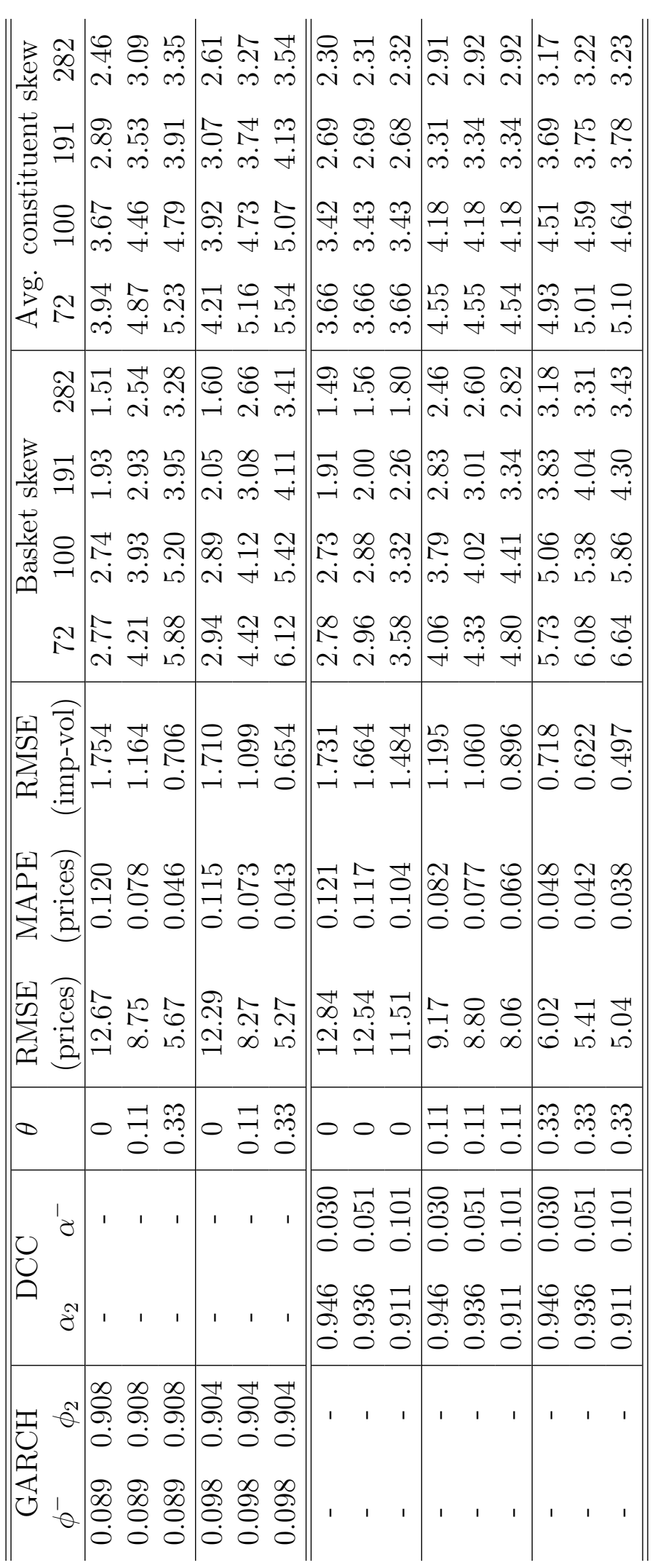

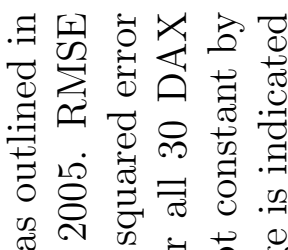

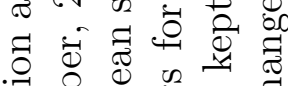

त्व द्व

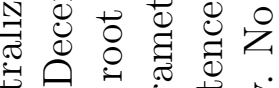

可

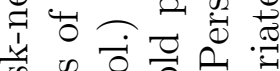



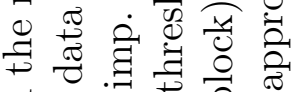

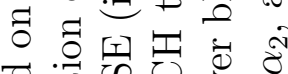

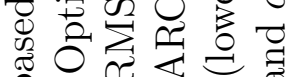

य

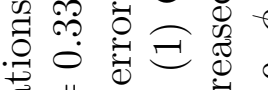

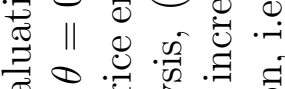

$>0 \begin{gathered}0 \\ 0 \\ 0\end{gathered}$

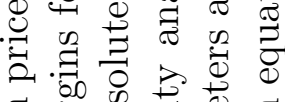

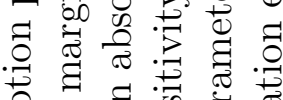

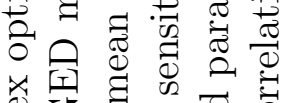

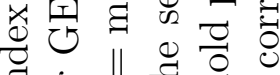

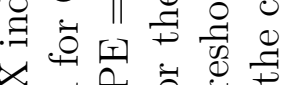

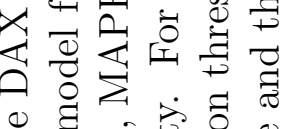

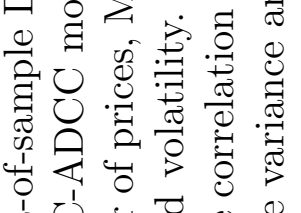

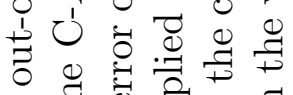

ธิ ए)

.

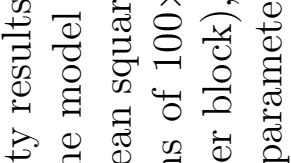

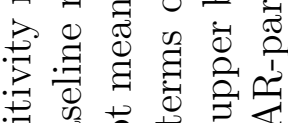

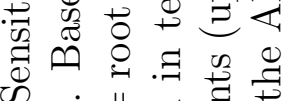

0
$\infty$
$\infty$

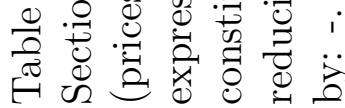




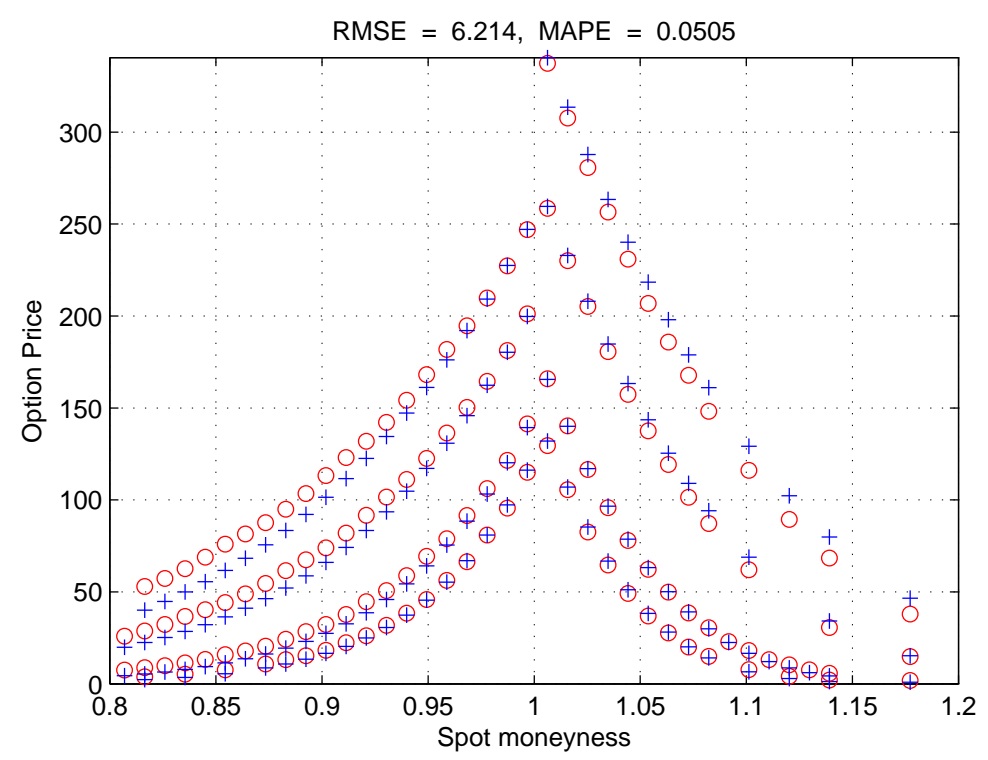

Figure 1: Price fit as of 7 December, 2005, for the GED based C-ADCC model with the Clayton copula for $\theta=0.33$; all other parameters as obtained by the CML/QML estimation. Crosses are model prices, circles correspond to observed prices of DAX index options. Option prices are displayed across spot moneyness, i.e. relative to the underlying asset. Out-of-themoney put prices appear for a spot moneyness less than one, out-of-the-money call prices for a spot moneyness larger than one. Options with 72 days to expiry appear as the lowest price function, options with 282 days to expiry as the top price function; options with 100 and 191 days to expiries appear in between. 

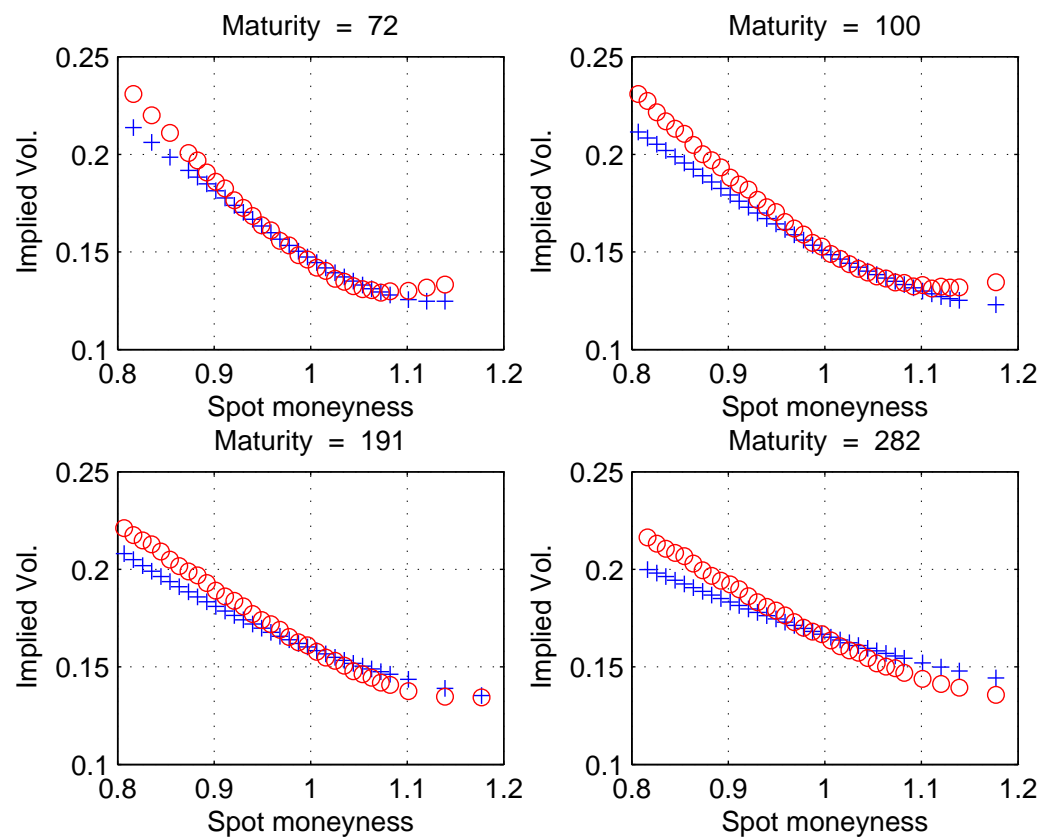

Figure 2: Implied volatility functions as of 7 December, 2005, for the GED based C-ADCC model with the Clayton copula for $\theta=0.33$; all other parameters as obtained by CML/QML estimation. Crosses are model implied volatilities, circles correspond to market implied volatilities of DAX index options. Implied volatility is displayed across spot moneyness, i.e. relative to the underlying asset, and maturity is given in calender days. 